\title{
The Obstruction to Fibering a Manifold over a Circle
}

\author{
F. T. FARRELL
}

Communicated by Andrew Wallace

Introduction. Let $M^{n}$ be a closed connected smooth manifold of dimension greater than five, and let $f: M \rightarrow S^{1}$ be a continuous map. The purpose of this paper is to give necessary and sufficient conditions for there to exist a smooth fiber map $\bar{f}: M \rightarrow S^{1}$ homotopic to $f$.

One condition is that $f_{*}$ is not the zero map, where $f_{*}: \Pi_{1}(M) \rightarrow \Pi_{1}\left(S^{1}\right)$. The necessity of this condition is seen by considering the homotopy exact sequence for a fiber space and remembering that the fiber should be a compact manifold. For convenience, we will assume that $f_{*}$ is onto. This condition corresponds geometrically to the fiber of a smooth fibration $\bar{f}$ being connected. By the 1-1 correspondence between homotopy classes of continuous maps of $M \rightarrow S^{1}$ and elements of $H^{1}(M ; Z)$, the above condition is equivalent algebraically to $f$ being an indivisible element of $H^{1}(M ; Z)$.

Let $G$ denote the kernel of $f_{*}$ and let $X$ be the covering space of $M$ corresponding to $G$. A second necessary condition is that $X$ must be the homotopy type of a finite C. W. complex. This follows since $X$ is the same homotopy type as the fiber of $\bar{f}$, if $\bar{f}$ exists.

Let $\left(N^{n-1}, v\right)$ be a closed framed submanifold of $M$ such that $(N, v)$ represents $f$ under the Pontryagin-Thom construction, $M_{N}$ denote the compact manifold obtained by "cutting" $M$ along $N . \partial M_{N}$ consists of two copies of $N$ which we denote by $N^{\prime}$ and $N^{\prime \prime}$. Hence, $M_{N}$ is a cobordism from $N^{\prime}$ to $N^{\prime \prime}$. The pair $\left(N^{n-1}, v\right)$ is called a splitting of $M$ with respect to $f$. Let $s$ be an integer smaller than $n-2$ and larger than 1 . Under the assumptions made thus far, we can always find a splitting $(N, v)$ such that $\left(M_{N}, N\right)$ has a handlebody decomposition consisting of only $s$ and $s+1$ dimensional handles.

The existence of a smooth fiber map $\bar{f}$ is equivalent to the existence of a splitting $(N, v)$ such that $M_{N}$ is diffeomorphic to $N \times[0,1]$. There are two obstructions to the existence of such a splitting. The first obstruction, denoted by $c(f)$, is an element of an abelian group $C(Z(G), \alpha)$ and vanishes if and only if there exists a splitting $(N, v)$ such that $\left(M_{N}, N\right)$ is an $h$-cobordism. (See Th. 5.2.) The second obstruction, denoted by $\tau(f)$, is defined if $c(f)=0$ and is an 
element of a quotient group of $W h(G) . \tau(f)$ vanishes if and only if there exists a splitting $(N, v)$ such that $M_{N}$ is diffeomorphic to $N \times[0,1]$. (See Th. 6.2.)

Our main theorem is the following:

Theorem 6.4. Let $M^{n}$ be a closed connected smooth manifold, $n \geqq 6$; let $f$ be an indivisible element from $H^{1}(M ; Z)$. Then there exists a smooth fiber map $\bar{f}: M \rightarrow S^{1}$ homotopic to $f$ if and only if the following three conditions hold.

$1^{\circ} X$ is the homotopy type of a finite C. W. complex.

$2^{\circ} c(f)=0$.

$3^{\circ} \tau(f)=0$.

We note that in the author's thesis (21), $c(f)$ was defined under the assumption that $X$ is dominated by a finite $\mathrm{C}$. W. complex. This is a weaker assumption than $1^{\circ}$ of Th. 6.4 .

Let us say a few words about the groups where the obstructions $c(f)$ and $\tau(f)$ lie. $C(Z(G), \alpha) \cong \widetilde{C}(Z(G), \alpha) \oplus \widetilde{K}_{0}(Z(G))$. (See Lemma 1.4.) If $Z(G)$ is Noetherian and the global dimension of $Z(G)$ is finite then $\tilde{C}(Z(G), \alpha)=0$. (See Th. 1.6.) The groups $\tilde{K}_{0}(Z(G))$ and $W h(G)$ have been extensively analyzed, in particular, when $G$ is a finitely generated abelian group. (See (13) and (14).) For certain groups $G, \tilde{C}(Z(G), \alpha), \tilde{K}_{0}(Z(G))$, and $W h(G)$ all vanish. In particular, this happens when $G$ is a finitely generated free abelian group. (To see this use Th. 1.6. on $\widetilde{C}(Z(G), \alpha)$ and see (13) for $W h(G)$ and $\tilde{K}_{0}(Z(G))$.) But, when $C(Z(G)$, $\alpha)$ and $W h(G)$ both vanish, conditions $2^{\circ}$ and $3^{\circ}$ of Th. 6.4 drop, and we are left with condition $1^{\circ}$ which is a purely homotopy theoretic condition. On the other hand, H. Bass and M. O. Murthy have shown that $\tilde{C}(Z(G)$, id) is not always zero. (See the remark following Th. 1.6.)

The results of this paper when $G=0$ have already been obtained by $\mathrm{W}$. Browder and J. Levine in (3). The reader would be well advised to familiarize himself with (3) before reading this paper. The first person to study the problem of when a manifold fibers a circle was J. Stallings. In (16) he solves this problem for 3 dimensional manifolds.

A version of our main theorem, Th. 6.4, is true in the case when $M$ is a manifold with a boundary, where the boundary already fibers a circle, and we wish to extend this fibration to the rest of $M$. We have restricted ourselves in this paper to the closed case in order to facilitate the discussion; but the interested reader is referred to the author's thesis (21) where the fibering theorem is proved in the case of a manifold with boundary. Also, see (22) for a statement of the result in the manifold with boundary case. In addition, Th. 6.4 is true in the piecewise linear category.

The interested reader will find in (21) a different proof that the obstruction $c(f)$ is well defined. This proof is perhaps useful for obtaining other results. He can also find in (22) an outline of some more recent methods, obtained by the author in 1968, which give an alternate approach to the problem of fibering a manifold over a circle. 
There will eventually appear a joint paper to be written with W. C. Hsiang which will discuss additional connections of the algebraic work of Bass and Murthy (14), and Bass, Heller, and Swan (1) with the theory of non-simply connected manifolds. Some of the results of this work have already been announced in (18) and (19). Also, Chapter III of this paper contains the proof of some theorems announced in Chapter III of (19).

This paper is a revised version of the author's doctoral dissertation, which was written under the direction of Professor Wu-chung Hsiang and submitted to Yale University in 1967 in partial fulfillment of the requirements for the Ph.D. degree. The author wishes to express his deep gratitude to Professor Hsiang for his valuable advice and constant encouragement which enabled the author to preservere to the completion of this research. Several times without Professor Hsiang's encouragement the author may have given up before carrying this research through to a successful conclusion. He also wishes to thank Professor Robert H. Szczarba for being a great source of knowledge and enthusiasm from which the author has continually drawn benefit.

Chapter I. The Grothendieck group of nilpotent matrices. The purpose of this chapter is to construct and derive the main properties of the group $C(R, \alpha)$. The major obstruction to a manifold fibering a circle lies in this group.

Let $R$ be a ring with identity, and $\alpha$ be an automorphism of $R$. Let $\mathcal{C}^{*}(R, \alpha)$ be the category whose objects are pairs $(M, f)$ where $M$ is a right $R$ module and $f$ is a $\alpha$ semi-linear endomorphism of $M$. (That is, $f(x r)=f(x) \alpha(r)$ where $x \varepsilon M$ and $r \varepsilon R$.) In this category the maps from $(M, f)$ to $(N, g)$ are the $R$ linear homomorphisms $\varphi: M \rightarrow N$ such that $g \circ \varphi=\varphi \circ f . \mathrm{C}^{*}(R, \alpha)$ is an abelian category. Let $\mathfrak{e}(R, \alpha)$ be the full subcategory of $\mathfrak{e}^{*}(R, \alpha)$ whose objects are pairs $(P, f)$ where $P$ is a finitely generated right projective $R$ module and $f$ is nilpotent. (That is, $f^{n}=0$ for some positive integer $n$.) Let $\tilde{\mathrm{e}}(R, \alpha)$ be the full subcategory of $\mathfrak{C}(R, \alpha)$ whose objects are pairs $(F, f)$ where $F$ is a stably free projective module and $P(R)$ be the category of all finitely generated projective modules. According to Bass, Heller, and Swan (See [1]), there exists an abelian group $C(R, \alpha)$ and a map $\sigma: \mathfrak{e}(R, \alpha) \rightarrow C(R, \alpha)$ which is universal with respect to the following properties.

$1^{\circ}$ If $F$ is free, then $\sigma(F, 0)=0$.

$2^{\circ}$ If $0 \rightarrow A_{2} \rightarrow A_{1} \rightarrow A_{0} \rightarrow 0$

is exact in $\mathcal{C}(R, \alpha)$, then $\sigma\left(A_{2}\right)-\sigma\left(A_{1}\right)+\sigma\left(A_{0}\right)=0$. In a similar fashion, the groups $\tilde{C}(R, \alpha)$ and $\tilde{K}_{0}(R)$ are defined from the categories $\tilde{\mathfrak{e}}(R, \alpha)$ and $\odot(R)$, respectively.

Next, we will give a more concrete definition of $C(R, \alpha)$, from which, we will show that $\sigma: \mathfrak{e}(R, \alpha) \rightarrow C(R, \alpha)$ is onto. To do this, we need a lemma which we now formulate.

Definition 1.1. A triangular object in $\mathcal{e}(R, \alpha)$ is a pair $(F, f)$ such that $F$ 
possesses a filtration $0=F_{0} \subseteq F_{1} \subseteq \cdots \subseteq F_{n}=F$ where each $F_{i} / F_{i-1}$ is free, and $f\left(F_{i}\right) \subseteq F_{i-1}$.

Lemma 1.2. Let $(K, k)$ be an object in $\mathfrak{e}^{*}(R, \alpha)$. Let $0=K_{0} \subseteq K_{1} \subseteq \cdots$ $\subseteq K_{m}=K$ be a filtration of $K$ by finitely generated submodules such that $k\left(K_{i}\right) \subseteq$ $\bar{K}_{i-1}$. Then there exists an exact sequence in $\mathfrak{e}^{*}(R, \alpha), 0 \rightarrow(L, \ell) \stackrel{a}{\rightarrow}(F, f) \stackrel{p}{\rightarrow}$ $(K, k) \rightarrow 0$ where $(F, f)$ is triangular with respect to a filtration $0=F_{0} \subseteq F_{1} \subseteq$ $\cdots \subseteq F_{m}=F$ such that $p\left(F_{i}\right)=K_{i}$.

Remark. If we define $L_{i}=q^{-1}\left(F_{i}\right)$, then $0=L_{0} \subseteq L_{1} \subseteq \cdots \subseteq L_{m}=L$ is a filtration of $L$ such that $0 \rightarrow L_{i} / L_{i-1} \rightarrow F_{i} / F_{i-1} \rightarrow K_{i} / K_{i-1} \rightarrow 0$ is exact.

The proof of Lemma 1.2 will be given at the end of chapter $\mathrm{I}$.

We now construct a group $C^{\prime}(R, \alpha)$, which we will show is isomorphic to $C(R, \alpha)$. The elements of $C^{\prime}(R, \alpha)$ are equivalence classes of isomorphism classes of objects from $\mathrm{e}(R, \alpha)$ where the equivalence relation is generated by the following elementary relations.

$1^{\prime \circ}$ If $F$ is free, then $(P, f) \sim(P \oplus F, f \oplus 0)$.

$2^{\prime \circ}$ If $0 \rightarrow\left(P_{2}, f_{2}\right) \rightarrow\left(P_{1}, f_{1}\right) \rightarrow\left(P_{0}, f_{0}\right) \rightarrow 0$ is exact in $\mathcal{e}(R, \alpha)$, then $\left(P_{1}, f_{1}\right)$ $\sim\left(P_{2} \oplus P_{0}, f_{2} \oplus f_{0}\right)$.

The group operation is defined by

$$
\{(P, f)\}+\{(Q, g)\}=\{(P \oplus Q, f \oplus g)\}
$$

(Here, $\{(P, f)\}$ denotes the equivalence class of the pair $(P, f)$.) With this definition $C^{\prime}(R, \alpha)$ is an abelian group. Only the existence of inverses is difficult to verify which we now proceed to verify.

The identity of the semi-group $C^{\prime}(R, \alpha)$ is $\{(0,0)\}$.

Lemma 1.3. Any triangular object is equivalent to $(0,0)$ under the equivalence relation generated by $1^{\prime \circ}$ and $2^{\prime \circ}$.

Proof. We proceed by induction on the length of the filtration by which the object is said to be triangular. If the length of the filtration is 1 , then Lemma 1.3 follows immediately from $1^{\prime \circ}$. Now suppose that Lemma 1.3 is true if the filtration is of length $n-1$ or less. Assume that our filtration is of length $m$. Consider the sequence

$$
0 \rightarrow\left(F_{n-1}, f / F_{n-1}\right) \rightarrow(F, f) \rightarrow\left(F_{n} / F_{n-1}, 0\right) \rightarrow 0 .
$$

Then $(F, f) \sim\left(F_{n-1} \oplus F_{n} / F_{n-1}, f / F_{n-1} \oplus 0\right)$ by $2^{\prime \circ}$. Also $\left(F_{n-1} \oplus F_{n} / F_{n-1}\right.$, $\left.f / F_{n-1} \oplus 0\right) \sim\left(F_{n-1}, f / F_{n-1}\right)$ by $1^{\prime \circ}$. And $\left(F_{n-1}, f / F_{n-1}\right) \sim(0,0)$ by the induction hypothesis. This proves Lemma 1.3.

If $(P, g)$ is an object from $\mathfrak{e}(R, \alpha)$ where $g$ is nilpotent of order $n$, let $K_{i}=$ image $g^{n-i}$. Then the filtration

$$
0=K_{0} \subseteq K_{1} \subseteq \cdots \subseteq K_{n}=P
$$


satisfies the hypothesis of Lemma 1.2. Hence there exists an exact sequence in $\mathcal{C}^{*}(R, \alpha), 0 \rightarrow(L, \ell) \rightarrow(F, f) \rightarrow(P, g) \rightarrow 0$. But since both $(F, f)$ and $(P, g)$ are in $\mathrm{e}(R, \alpha)$, this implies that $(L, \ell)$ is in $\mathrm{e}(R, \alpha)$. By $2^{\circ \circ},(L \oplus P, \ell \oplus g) \sim$ $(F, f)$. Since $(F, f)$ is triangular, $(F, f) \sim(0,0)$ by Lemma 1.3. Therefore $\{(L, \ell)\}$ is the inverse of $\{(P, g)\}$ in $C^{\prime}(R, \alpha)$. Hence $C^{\prime}(R, \alpha)$ is a group.

It is easy to see that $C^{\prime}(R, \alpha)$ is universal with respect to properties $1^{\circ}$ and $2^{\circ}$ used in defining $C(R, \alpha)$. Hence $C^{\prime}(R, \alpha)$ is naturally isomorphic to $C(R, \alpha)$. Thus we have a new construction for $C(R, \alpha)$ from which it is clear that $\sigma$ : $\mathfrak{e}(R, \alpha) \rightarrow C(R, \alpha)$ is onto. We could give similar explicit constructions for the groups $\tilde{C}(R, \alpha)$ and $\tilde{K}_{0}(R)$. In particular the maps $\sigma: \tilde{\mathfrak{e}}(R, \alpha) \rightarrow \tilde{C}(R, \alpha)$ and $\sigma: \mathcal{P}(R) \rightarrow \tilde{K}_{0}(R)$ are both onto.

Next we show that $C(R, \alpha)$ splits as a direct sum of $\tilde{C}(R, \alpha)$ and $\tilde{K}_{0}(R)$. There is an additive functor $\mathcal{F}: \mathcal{C}(R, \alpha) \rightarrow \mathcal{P}(R)$ which sends $(P, f)$ to $P$. The functor $\mathcal{F}$ induces a map $\bar{F}: C(R, \alpha) \rightarrow \tilde{K}_{0}(R)$. There is a functor $\mathcal{J}: \mathcal{P}(R) \rightarrow$ $\mathfrak{C}(R, \alpha)$ given by sending $P$ to $(P, 0) . J$ induces a map $\bar{J}: \tilde{K}_{0}(R) \rightarrow C(R, \alpha)$. $\bar{J}$ is a splitting of $\bar{F}$. Let $I: \tilde{\mathfrak{C}}(R, \alpha) \rightarrow \mathfrak{C}(R, \alpha)$ denote the inclusion functor. Then $I$ induces a homomorphism $\bar{I}: \tilde{C}(R, \alpha) \rightarrow C(R, \alpha)$. It is easily seen that $\bar{F} \circ \bar{I}=0$. If $(P, f)$ is an object in $\mathcal{C}(R, \alpha)$ then there exists a $Q$ in $\mathcal{P}(R)$ such that $P \oplus Q$ is free. Hence $\sigma(P, f)=\bar{I} \sigma(P \oplus Q, f \oplus 0)-\bar{J} \sigma(Q, 0)$. Therefore image $\bar{I}$ equals kernel $\bar{F}$. If to the object $(P, f)$ in $\mathcal{C}(R, \alpha)$ we assign $\sigma(P \oplus Q$, $f \oplus 0) \varepsilon \tilde{C}(R, \alpha)$, it is easily checked that we obtain a well defined map of $\mathcal{C}(R, \alpha)$ to $\tilde{C}(R, \alpha)$. This map satisfies properties $1^{\circ}$ and $2^{\circ}$, and hence defines a map of $C(R, \alpha)$ to $\tilde{C}(R, \alpha)$ which is seen to be a right inverse to $\bar{I}$. Hence we have the following lemma.

Lemma 1.4. $C(R, \alpha)$ splits as a direct sum of $\tilde{C}(R, \alpha)$ and $\tilde{K}_{0}(R)$.

The group $\tilde{K}_{0}(R)$ has been extensively studied. (See, for example, [14].) We next obtain a result stating that $\widetilde{C}(R, \alpha)=0$ for a certain class of rings $R$.

Definition 1.5. A ring $R$ is said to be right regular if $R$ is right Noetherian, and every finitely generated right $R$ module has a projective resolution of finite length.

Theorem 1.6. If $R$ is right regular, then $\tilde{C}(R, \alpha)=0$.

Remark. The rings which will concern us are integral group rings, denoted by $Z(G)$ where $G$ is the group. As an example of Theorem 1.6, let $G=Z^{n}$, the free abelian group of rank $n$, and $R=Z(G)$. This ring is right regular, and hence $\tilde{C}\left(Z\left(Z^{n}\right), \alpha\right)=0$. Also $\tilde{K}_{0}\left(Z\left(Z^{n}\right)\right)=0$ (see [13], page 419, ex. 6) and therefore $C\left(Z\left(Z^{n}\right), \alpha\right)=0$. On the other hand, Bass and Murthy have shown that $\tilde{C}(Z(G), i d) \neq 0$ when $G$ is a finitely generated abelian group of rank $\geq 1$ and the order of the torsion subgroup of $G$ has a square factor. This can be derived from the results of [1] and [14].

Proof of Theorem 1.6. Let $(P, g)$ be an object from $\tilde{\mathfrak{e}}(R, \alpha)$. Then $g$ is nilpotent of some order $n$. Let $K_{i}=$ image $g_{n}{ }^{-i}$; then $0=K_{0} \subseteq K_{1} \subseteq \cdots \subseteq K_{n}=P$ 
is a filtration of $P$ satisfying the hypothesis of Lemma 1.2. Hence there exists an exact sequence in $\mathcal{C}^{*}(R, \alpha), 0 \rightarrow\left(P_{1}, g_{1}\right) \rightarrow(F, f) \rightarrow(P, g) \rightarrow 0$. Since $(F, f)$ is triangular, $\sigma\left(P_{1}, g_{1}\right)=-\sigma(P, g)$. But by the remark following Lemma 1.2, we see that there exists a filtration of $P_{1}, 0=L_{0} \subseteq L_{1} \subseteq \cdots \subseteq L_{n}=P_{1}$, such that $g\left(L_{i}\right) \subseteq L_{i-1}$ and $0 \rightarrow L_{i} / L_{i-1} \rightarrow F_{i} / F_{i-1} \rightarrow K_{i} / K_{i-1} \rightarrow 0$ is exact. Since $R$ is Noetherian, we see that $L_{i} / L_{i-1}$ is finitely generated. If $M$ is a right $R$ module, let $d(M)$ denote the length of a projective resolution of $M$ of minimal length. Then $d\left(L_{i} / L_{i-1}\right)=\max \left(1, d\left(K_{i} / K_{i-1}\right)-1\right)$. Since $R$ is Noetherian, each $L_{i}$ is finitely generated, and hence $\left(P_{1}, g_{1}\right)$ satisfies the hypothesis of Lemma 1.2 with respect to the filtration $0=L_{n} \subseteq L_{1} \subseteq \cdots \subseteq L_{n}=P_{1}$. Let $m=$ $\max _{1 \leqq i \leqq n} d\left(K_{i} / K_{i-1}\right)$. Then after $m$ applications of Lemma 1.2 , we obtain an object $\left(P_{m}, g_{m}\right)$ of $\tilde{\mathcal{e}}(R, \alpha)$ such that $\sigma\left(P_{m}, g_{m}\right)=(-1)^{m} \sigma(P, g)$ and a filtration $0=S_{0} \subseteq S_{1} \subseteq \cdots \subseteq S_{n}=P_{m}$ such that each $S_{i} / S_{i-1}$ is an object from $\odot(R)$. By application of the defining property $2^{\circ}$ for $\tilde{C}(R, \alpha)$, we see that $\sigma\left(P_{m}\right.$, $\left.g_{m}\right)=\sum_{i=1}^{n} \sigma\left(S_{i} / S_{i-1}, 0\right)=\sigma\left(\oplus_{i=1}^{n}\left(S_{i} / S_{i-1}\right), 0\right)=\sigma\left(P_{m}, 0\right)=0$, since $P_{m}$ is stably free, and hence $\tilde{C}(R, \alpha)=0$.

The $\alpha$-twisted polynomial ring $R_{\alpha}[t]$ is defined as follows. Additively, $R_{\alpha}[t]=$ $R[t]$. Multiplicatively, for $f=a t^{n}$ and $g=b t^{m}$ two monomials, $f \circ g=a \alpha^{n}(b) t^{n+m}$. Similarly, we define the $\alpha$-twisted finite Laurentz series ring $R_{\alpha}[T]=R_{\alpha}\left[t, t^{-1}\right]$.

Lemma 1.7. Let $P$ be a module over $R_{\alpha}[t]$ such that $P t^{n}=0$ for some $n$. Suppose that $0 \rightarrow X \rightarrow Y \rightarrow P \rightarrow 0$ is exact in $R_{\alpha}[t]$ where $X$ and $Y$ are finitely generated projective $R_{\alpha}[t]$ modules. Then $P$ is projective when considered as a module over $R$.

Remark $1^{\circ}$. Right multiplication by $t$ is an $\alpha^{-1}$ semi-linear endomorphism of $P$, and hence it follows from Lemma 1.7 that $(P, t) \varepsilon \mathcal{e}\left(R, \alpha^{-1}\right)$.

Remark 2. Lemma 1.7 remains true when the map from $X \rightarrow Y$ is only $\alpha^{i}$ semi-linear.

The proof of Lemma 1.7 will be postponed until the end of this chapter.

We next define a map $p: K_{1}\left(R_{\alpha}\left[t, t^{-1}\right]\right) \rightarrow C\left(R, \alpha^{-1}\right)$. Let $f:\left(R_{\alpha}[T]\right)^{n} \rightarrow$ $\left(R_{\alpha}[T]\right)^{n}$ represent an element in $K_{1}\left(R_{\alpha}\left[t, t^{-1}\right]\right)$. Then, $R_{t^{m}} \circ f:\left(R_{\alpha}[t]\right)^{n} \rightarrow$ $\left(R_{\alpha}[t]\right)^{n}$ for some positive integer $m$. (Here $R_{a}$ denotes right multiplication by $a$.) Let $M\left(\mathfrak{R}_{t^{m}} \circ f\right)=\left(R_{\alpha}[t]\right)^{n} / \mathcal{R}_{t^{m}} \circ f\left(\left(R_{\alpha}[t]\right)^{n}\right)$. By Lemma 1.7 we see that $\left(M\left(\mathbb{R}_{t^{m}} \circ\right.\right.$ $\left.f), \Omega_{t}\right) \varepsilon \mathfrak{e}\left(R, \alpha^{-1}\right)$. Define $p: K_{1}\left(R_{\alpha}[T]\right) \rightarrow C\left(R, \alpha^{-1}\right)$ by $p(f)=\sigma\left(M\left(R_{t} \circ f\right)\right.$, $\left.R_{t}\right)$.

Remark. The map $p$ and Lemma 1.7 were announced in [19]. They represent joint work of the author and W. C. Hsiang.

If $f: X \rightarrow Y$ is a linear or semi-linear map, we define $M(f)=Y / f(X)$. For the sake of brevity, if $f$ denotes the map from $X \rightarrow Y$ in Lemma 1.7 then $M(f)$ will also be used to denote the object $\left(M(f), \Re_{t}\right) \varepsilon \mathfrak{e}\left(R, \alpha^{-1}\right)$.

We use the following lemma to show that $p$ is well defined. 
Lemma 1.8. If $P_{1}, P_{2}$, and $P_{3}$ are modules over a ring $R$, and $f_{1}: P_{1} \rightarrow$ $P_{2}, f_{2}: P_{2} \rightarrow P_{3}$ are either linear or semi-linear maps, where $f_{2}$ is a monomorphism, then the following sequence is exact:

$$
0 \rightarrow f_{2}\left(M\left(f_{1}\right)\right) \rightarrow M\left(f_{2} \circ f_{1}\right) \rightarrow M\left(f_{2}\right) \rightarrow 0 .
$$

Proof. Lemma 1.8 follows immediately by considering the triple $f_{2}\left(f_{1}\left(P_{1}\right)\right) \subset$ $f_{2}\left(P_{2}\right) \subset P_{3}$.

Applying Lemma 1.8 to the maps

$$
\left(R_{\alpha}[t]\right)^{n} \stackrel{Q_{t}}{\longrightarrow}\left(R_{\alpha}[t]\right)^{n} \stackrel{Q_{t m^{\circ} f}}{\longrightarrow}\left(R_{\alpha}[t]\right)^{n},
$$

we obtain that

$$
0 \rightarrow \mathbb{R}_{t m} \circ f\left(M\left(\mathbb{R}_{t}\right)\right) \rightarrow M\left(\mathbb{R}_{t m} \circ f \circ \mathbb{R}_{t}\right) \rightarrow M\left(\mathbb{R}_{t m} \circ f\right) \rightarrow 0 .
$$

is exact. (Here $M$ is considered as a module over $R_{\alpha}[t]$.) Since $f$ is linear, $f \circ$ $\mathbb{R}_{t}=\Omega_{t} \circ f$. Hence $\sigma\left(\Omega_{t m} \circ f\left(M\left(\mathbb{R}_{t}\right)\right)\right)+\sigma\left(M\left(\mathbb{R}_{t m} \circ f\right)\right)=\sigma\left(M\left(\mathbb{R}_{t m+1} \circ f\right)\right)$. Since $R_{t m}$ is $\alpha^{-m}$ semi-linear

$$
\sigma\left(\Omega_{t^{m}} \circ f\left(M\left(\Omega_{t}\right)\right)\right)=\alpha_{*}^{-m}\left(\sigma\left(M\left(\Omega_{t}\right)\right)\right) .
$$

(The automorphism $\alpha$ induces a map $\alpha_{*}: C(R, \alpha) \rightarrow C(R, \alpha)$ in the obvious manner.) But $M\left(\Omega_{t}\right)=\left(R^{n}, 0\right)$. Hence $\sigma\left(M\left(R_{t}\right)\right)=0$. Therefore

$$
\sigma\left(M\left(R_{t m} \circ f\right)\right)=\sigma\left(M\left(R_{t m+1} \circ f\right)\right),
$$

and hence $p$ is well defined from

$$
G L\left(R_{\alpha}[T]\right) \rightarrow C(R, \alpha) .
$$

Notice that if we apply Lemma 1.8 to the maps

$$
\left(R_{\alpha}[t]\right)^{n} \stackrel{Q_{t m^{\circ} f}}{\longrightarrow}\left(R_{\alpha}[t]\right)^{n} \stackrel{a_{t}}{\longrightarrow}\left(R_{\alpha}[t]\right)^{n},
$$

we obtain that $\sigma\left(M\left(\Omega_{t m+1} \circ f\right)\right)=\alpha_{*}^{-1}\left(\sigma\left(M\left(\Omega_{t m} \circ f\right)\right)\right)$. Hence we obtain the following corollary.

Corollary 1.9. $\alpha_{*}(g(f))=p(f)$.

Now we complete the proof that $p$ is well defined. Let $f, g:(R[T])^{n} \rightarrow(R[T])^{n}$ be elements of $G L(R[T])$. If we apply Lemma 1.8 to $f$ and $g$, we obtain that

$$
p(f \circ g)=p(f)+p(g), \text { and hence } p: G L(R[T]) \rightarrow C\left(R, \alpha^{-1}\right)
$$

is a group homomorphism. But, $C\left(R, \alpha^{-1}\right)$ is abelian, and hence $p([G L(R[T])$, $G L(R[T])])=0$. Therefore, $p$ defines a homomorphism $p: K_{1}(R[T]) \rightarrow C\left(R, \alpha^{-1}\right)$. (Here we use the fact that $K_{1}(R[T])=G L(R[T]) /[G L(R[T]), G L(R[T])]$.) That is, $p$ is well defined.

We are particularly interested in the case where $R$ is the integral group ring of a group $G$. Let $\alpha$ be an automorphism of $G$, and let $G \odot_{\alpha} Z$ denote the semidirect product of $G$ with $Z$ via $\alpha$. Then $\alpha$ induces an automorphism of $Z(G)$ 
which we also denote by $\alpha$. Since $Z(G \odot Z) \cong Z(G)_{\alpha}[T], p: K_{1}\left(Z\left(G \odot_{\alpha} Z\right)\right) \rightarrow$ $C\left(Z(G), \alpha^{-1}\right)$. One easily sees that $p$ factors through $W h\left(G \odot_{\alpha} Z\right)$. Therefore, we obtain a map

$$
p: W h(G \underset{\alpha}{\odot} Z) \rightarrow C\left(Z(G), \alpha^{-1}\right) .
$$

We end this chapter with the proofs of Lemmas 1.2 and 1.7.

Proof of Lemma 1.2. The proof is by induction on $n$. For $n=1$, since $K$ is finitely generated we can find a map $p: F \rightarrow K \rightarrow 0$ where $F$ is a finitely generated free module. Then $0 \rightarrow($ ker $p, 0) \rightarrow(F, 0) \stackrel{p}{\rightarrow}(K, 0) \rightarrow$ is the desired sequence. Assume that the lemma is true for $n-1$. Therefore, there exists a map

$$
p:\left(F_{n-1}, f\right) \rightarrow\left(K_{n-1}, k\right) \rightarrow 0
$$

satisfying the conclusion of Lemma 1.2 . Since $K_{n} / K_{n-1}$ is finitely generated, there exists a finitely generated free module $Q$ and a map $q: Q \rightarrow K_{n} / K_{n-1} \rightarrow 0$. This lifts to a map

$$
\hat{q}: Q \rightarrow K_{n}=K . \text { Let } F=F_{n-1} \oplus Q
$$

and extend the definition of $p$ to $F$ by the use of $\hat{q}$. Then, $p: F \rightarrow K \rightarrow 0$. Consider the following diagram.

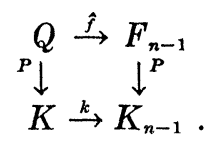

Since $Q$ is free and $k \circ p$ is $\alpha$ semi-linear, it is easily seen that $k \circ p$ lifts to an $\alpha$ semi-linear map $\hat{f}$. Extend from $F_{n-1}$ to $F$ by the use of $\hat{f}$. Then the pair $(F, f)$ and the map $p$ clearly satisfy the conclusion of Lemma 1.2. Let $L=\operatorname{ker} p$, $\ell=f / \operatorname{ker} p$, and $q:(L, \ell) \rightarrow(F, f)$ be the inclusion map. This proves Lemma 1.2.

In order to prove Lemma 1.7, we need the following result.

Lemma 1.10. Let $A_{i}$ and $B_{i}, i=1,2,3$, be modules over a ring $R$. Let $f_{i}$ : $A_{i} \rightarrow B_{i}$ be linear or semi-linear maps. Assume that the following diagram commutes:

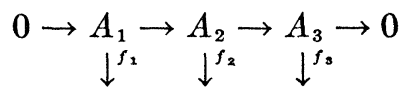

$$
\begin{aligned}
& 0 \rightarrow B_{1} \rightarrow B_{2} \rightarrow B_{3} \rightarrow 0
\end{aligned}
$$

where the rows are exact. Assume also that $f_{3}$ is a monomorphism. Then,

$$
0 \rightarrow M\left(f_{1}\right) \rightarrow M\left(f_{2}\right) \rightarrow M\left(f_{3}\right) \rightarrow 0
$$

is exact.

Proof. Do the obvious diagram chasing. 
Suggestion. To understand the proof of Lemma 1.7, it may be helpful to look at the proof of Theorem 8, part b, of [23].

Proof of Lemma 1.7. Since $P t^{n}=0$, if we consider $X, Y$ and $P$ as modules over $R_{\alpha^{n}}[s]$ where $s=t^{n}$, we see that $X$ and $Y$ are finitely generated projective $R_{\alpha^{n}}[s]$ modules and that $P s=0$. If we consider $X$ and $Y$ as $R$ modules, then they are projective. We obtain a new $R_{\alpha^{n}}[s]$ module structure on $X$ and $Y$ by defining $X s=Y s=0$. Denote these modules by $X^{\prime}$ and $Y^{\prime}$.

Some Notation. $1^{\circ}$. If $M$ is a module over $R$, then $M_{\alpha}$ is a new module over $R$ defined as follows: Additively, $M_{\alpha}=M$. Multiplicatively, if $x \varepsilon M_{\alpha}$ and $r \varepsilon R$, then $x \cdot r=x \alpha(r)$ (the right hand side being multiplication in $M$ ).

$2^{\circ}$. If $r$ is an element from a ring $R$ then $\mathscr{L}_{r}$ denotes left multiplication by $r$. $3^{\circ}$. In the hypothesis of Lemma 1.7, let $f$ denote the map from $X$ to $Y$.

Since $X$ is a projective $R$ module, $X_{\alpha^{n}}$ is also projective. Hence,

$$
0 \rightarrow X_{\alpha^{n}} \bigotimes_{R} R_{\alpha^{n}}[s] \stackrel{i d \otimes \mathcal{L}_{s}}{\longrightarrow} X \underset{R}{\bigotimes} R_{\alpha^{n}}[s] \rightarrow X^{\prime} \rightarrow 0
$$

is a resolution of $X^{\prime}$ by projective $R_{\alpha^{n}}[s]$ modules. Denote the map id $\otimes \mathscr{L}$. by $g$. Consider the following diagram of $R_{\alpha^{\star}}[s]$ modules

$$
\begin{aligned}
& \begin{array}{cc}
0 & 0 \\
\downarrow & \downarrow \\
\alpha_{\alpha^{n}} \underset{R}{\searrow} R_{\alpha^{n}}[s] & X
\end{array} \\
& X \underset{R}{\otimes} \stackrel{\downarrow}{R}_{\alpha^{n}}[s] \quad \downarrow^{f} \quad Y \\
& 0 \rightarrow \stackrel{\downarrow}{X^{\prime}} \stackrel{f}{\rightarrow} Y^{\prime} \rightarrow \stackrel{\downarrow}{\perp} \rightarrow 0
\end{aligned}
$$

It is a standard result from homological algebra that there exists a map

$$
\eta: X \rightarrow X \underset{R}{\otimes} R_{\alpha^{n}}[s]
$$

such that $Y^{\prime}$ is the cokernel of the map

$$
F:\left(X_{\alpha^{n}} \otimes_{R} R_{\alpha^{n}}[s]\right) \oplus X \rightarrow\left(X \otimes_{R} R_{\alpha^{n}}[s]\right) \oplus Y
$$

defined by $F(a, b)=(g(a)+\eta(b), f(b))$. Let $\bar{X}$ and $\bar{Y}$ denote

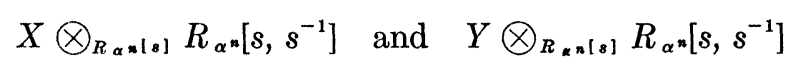

respectively. Let $\bar{f}$ denote $f \otimes i d: \bar{X} \rightarrow \bar{Y}$. Then, $X \subset \bar{X}, Y \subset \bar{Y}$ and $\bar{f}$ is an extension of $f$. Since $P s=0, \bar{f}$ is an isomorphism. Since $Y$ is finitely generated, 
there exists an integer $m$ such that $\Omega_{s^{m}} \circ \bar{f}^{-1}(Y) \subset X$. Define $\eta^{\prime}: Y \rightarrow X \otimes_{\boldsymbol{R}}$ $R_{\alpha}[s]$ by $\eta^{\prime}=\eta \circ R_{s^{m}} \circ \bar{f}^{-1}$. Define $F^{\prime}:\left(X \otimes_{R} R_{\alpha^{n}}[s]\right) \oplus Y \rightarrow\left(X \otimes_{R} R_{\alpha^{n}}[s]\right) \oplus Y$ by $F^{\prime}(x, y)=\left(x s^{m}+\eta^{\prime}(y), y s^{m}\right)$. Then $F^{\prime} \circ(g \oplus f)=\mathcal{R}_{\mathrm{s} m} \circ F$. $F^{\prime}$ and $\mathcal{R}_{\mathrm{s} m}$ are both monomorphisms, and hence by Lemma 1.8 we obtain the following two exact sequences.

$\left.1^{\circ}\right) 0 \rightarrow F^{\prime}(M(g \oplus f)) \rightarrow M\left(F^{\prime} \circ(g \oplus f)\right) \rightarrow M\left(F^{\prime}\right) \rightarrow 0$

$\left.2^{\circ}\right) 0 \rightarrow \mathbb{R}_{s m}(M(F)) \rightarrow M\left(\mathcal{R}_{s m} \circ F\right) \rightarrow M\left(\mathcal{R}_{s m}\right) \rightarrow 0$

It is easily seen that $M\left(\Omega_{s}\right)$ is a projective $R$ module. Hence by $2^{\circ}, M\left(\Omega_{s} \circ F\right)$ $\cong M\left(R_{s}\right) \oplus R_{s^{m}}\left(Y^{\prime}\right)$ as $R$ modules. But, $Y^{\prime}$ is projective as an $R$ module and hence $M\left(\Omega_{s m} \circ F\right)=M\left(F^{\prime} \circ(g \oplus f)\right)$ is projective. Applying Lemma 1.10 to $F^{\prime}$, we obtain the exact sequence

$$
0 \rightarrow M\left(\mathcal{R}_{s^{m}}\right) \rightarrow M\left(F^{\prime}\right) \rightarrow M\left(\mathcal{R}_{s m}\right) \rightarrow 0 .
$$

Hence, $M\left(F^{\prime}\right)$ is a projective $R$ module. Therefore, by $1^{\circ}$, we see that $F^{\prime}(M(g \oplus f))$ is $R$ projective. Since $F^{\prime}$ is $\alpha^{m n}$ semi-linear, $M(g \oplus f)$ is $R$ projective. But clearly, $M(g \oplus f) \cong M(g) \oplus M(f)=X^{\prime} \oplus P$, and hence $P$ is a projective $R$ module. This completes the proof of Lemma 1.7.

Chapter II. Geometric simplification of the fibering problem. Let $M^{n}$ be a closed connected $C^{\infty}$ manifold of dimension $\geqq 6$, and let $f$ be a continuous map of $M$ into $S^{1}$. Suppose that $f_{*}: \Pi_{1}(M) \rightarrow \Pi_{1}\left(S^{1}\right)$ is onto.

Remark. The homotopy classes of maps of a space $X$ into $S^{1}$ correspond in a one to one fashion to elements of $H^{1}(X ; Z)$. Under this correspondence, the above condition is equivalent to $f$ being an indivisible element of $H^{1}(X ; Z)$.

Our problem is to determine when there exists a smooth fiber map $\bar{f}: M \rightarrow S^{1}$ such that $\bar{f}$ is homotopic to $f$. (See [3] for the definition of smooth fiber map.)

Definition 2.1. A pair $(N, \nu)$ will be called a splitting of $M$ with respect to $f$ if $N$ is a compact $n-1$ dimensional submanifold of $M$ and $\nu$ is a framing for the normal bundle of $N$ such that under the Pontryagin-Thom construction $(N, \nu)$ represents $f$.

When no confusion can result we denote a splitting merely by $N$.

Definition 2.2. $W$ is an elementary cobordism of dimension $s$ from a splitting $(N, \nu)$ to a splitting $\left(N^{\prime}, \nu^{\prime}\right)$ if $W$ is a $n$ dimensional compact submanifold of $M$ such that $\partial W=N \cup N^{\prime}$, and the vector field $\nu$ points into $W$ while $\nu^{\prime}$ points out of $W$. Also, we require $W$ to be diffeomorphic to $N \times[0,1] \cup_{\varphi}$ $D^{s} \times D^{n-s}$, where $\varphi$ is a diffeomorphism of $S^{s-1} \times D^{n-s}$ into $N \times 1$.

Remark. In the literature, the passage from $N$ to $N^{\prime}$ via $W$ is referred to as exchanging an $s$-dimensional handle from one side of $N$ to the other. $N$ will be called the left side of $W$ and $N^{\prime}$ the right side.

We now embark on a program for improving splittings. If we start with an arbitrary $N$ (splittings always exist by the Pontryagin-Thom construction), 
we can pass to a new splitting $N^{\prime}$, where $N^{\prime}$ is connected, by a sequence of elementary cobordisms of dimension 1. This is done explicitly in [3] on page 157. Here we use the fact that $f_{*}: \Pi_{1}(M) \rightarrow \Pi_{1}\left(S^{1}\right)$ is onto.

Let $G=\operatorname{ker} f_{*}$. Since $G$ is a normal subgroup, we will generally omit considerations of base point. Let $X$ be the covering space of $M$ corresponding to $G$. A necessary condition for the existence of a smooth fiber map $\bar{f}$ homotopic to $f$ is that $X$ be dominated by a finite $\mathrm{C}$. W. complex. Under this single assumption we will see how much improvement of splittings we can effect. We have $N$ connected, and we wish next to obtain the situation where $i_{*}: \Pi_{1}(N) \rightarrow \Pi_{1}(M)$ is a monomorphism with image $G$ ( $i$ denotes the inclusion of $N$ into $M$ ). First, we need the following algebraic lemma.

Lemma 2.3. Let $A, B, C$, and $D$ be groups, and let $f: A \rightarrow B$ and $g: C \rightarrow B$ be homomorphisms such that kernel $D=k e r g$ and $g$ is onto. Assume that $A$ and $C$ are finitely generated, and that $D$ is the normal closure in $C$ of a finite set of elements. Let $A \circ C$ denote the free product of $A$ and $C$. Let $h: A \circ C \rightarrow B$ be the homomorphism induced by $f$ and $g$. Then $h$ is onto and its kernel is the normal closure in $A \circ C$ of a finite set of elements.

For a proof of Lemma 2.3 see [5] page 4.

Since $X$ is dominated by a finite C. W. complex, we have that $\Pi_{1}(X) \cong G$ is finitely presented (see [6] Lemma 1.3.). Let $F$ be a free group on $m$ generators $a_{1}, \cdots, a_{m}$, and $g$ a homomorphism of $F$ into $G$ whose kernel $K$ is the normal closure in $F$ of a finite set of elements. Let $x$ be a point inside a tubular neighborhood of $N$ but not on $N$. Let $L_{1}, \cdots, L_{m}$ be circles embedded in $M$ such that each $L_{i}$ meets $N$ transversally and $L_{i} \cap L_{i}=x$ if $i \neq j$. We pick $L_{i}$ so that $L_{i}$ represents $g\left(a_{i}\right)$. Since under the Pontryagin-Thom construction $(N, \nu)$ represents $f$ and since $f_{*}\left(g\left(a_{i}\right)\right)=0$, we see that the intersection number of $L_{i}$ with $N$ is 0 . Hence, after exchanging a finite number of handles of dimension one, we can obtain the situation where $L_{i}$ does not meet $N$. We do this for each $i$, finally obtaining the situation where the bouquet of circles $L_{1}, \cdots, L_{m}$ is disjoint from our new splitting $N^{\prime}$. Next, by exchanging one dimensional handles $W_{1}, \cdots, W_{m}$ such that the core of $W_{i}$ is homotopic to $L_{i}$, we obtain a connected splitting $N^{\prime \prime}$ such that by van Kampen's theorem $\Pi_{1}\left(N^{\prime \prime}\right) \cong \Pi_{1}\left(N^{\prime}\right) \circ F$, and the inclusion map $i_{*}^{\prime \prime}: \Pi_{1}\left(N^{\prime \prime}\right) \rightarrow G \subseteq \Pi_{1}(M)$ is induced from $\ell_{*}^{\prime}: \Pi_{1}\left(N^{\prime}\right) \rightarrow G$ and $g: F \rightarrow G \rightarrow 0$. Hence, by the algebraic lemma above, the kernel of $i_{*}^{\prime \prime}$ is the normal closure in $\Pi_{1}\left(N^{\prime \prime}\right)$ of a finite set of elements. Now, by exchanging a finite sequence of 2 dimensional handles, (see [7], the proof of Lemma 3.1), we obtain a connected splitting $N^{\prime \prime \prime}$ such that $i_{*}^{\prime \prime \prime}: \Pi_{1}\left(N^{\prime \prime \prime}\right) \rightarrow \Pi_{1}(M)$ is a monomorphism whose image is $G$.

With $N$ thus improved, choose a lifting $\hat{N}$ of $N$ to $X$. Then $\hat{N}$ divides $X$ into two connected components which we denote by $A$ and $B$ (see [3] Sec. 3.1.). The framing $\nu$ also lifts to a framing $\hat{\nu}$. Let $B$ denote the component into which $\hat{\nu}$ points. When no confusion can result, we will use $N$ and $\nu$ to denote $\hat{N}$ and $\hat{\nu}$. Let $T$ denote that generator of the group of covering transformations of $X$ such 
that $A \subset T(A), \tilde{X}$ the universal covering space of $X$, and $p: \widetilde{X} \rightarrow X$ the covering projection. $\widetilde{A}, \widetilde{B}$ and $\widetilde{N}$ will denote $p^{-1}(A), p^{-1}(B)$, and $p^{-1}(N)$, respectively. Since the inclusion map of $N$ into $X$ induces an isomorphism on fundamental groups, we see that $\tilde{N}$ is connected and simply connected. Since $N \subset A \subset X$, we see that the inclusion of $A$ into $X$ induces an epimorphism on fundamental groups, and hence $\widetilde{A}$ is connected. Likewise, $\widetilde{B}$ is connected. By applying van Kampen's theorem to $\tilde{N}, \tilde{A}, \widetilde{B}$ and $\tilde{X}$ we see that $\tilde{A}$ and $\widetilde{B}$ are both simply connected. Hence, the inclusion maps of $A$ and $B$ into $X$ induce isomorphisms on fundamental groups.

Consider the groups $H_{i}(\tilde{X}, \tilde{A} ; Z)$. Identify $G$ with the group of covering transformations of $\tilde{X}$. Then $H_{i}(\tilde{X}, \tilde{A} ; Z)$ becomes a right $Z(G)$ module via the action of $G$ on $\tilde{X}$. We denote these modules by $H_{i}(X, A ; Z(G))$. Since $X$ and $N$ are dominated by finite C. W. complexes, and the inclusions of $A$ and $B$ into $X$ induce isomorphisms on fundamental groups, and $A, B, N$ and $X$ are connected, we have that $A$ and $B$ are both dominated by finite C. W. complexes (see [2] Complement 6.6.). It also follows that $H_{0}(X, A ; Z(G))$ and $H_{1}(X, A ; Z(G))$ are both zero.

Definition 2.4. A splitting $N$ is s-connected if $N$ is connected; $i_{*}$ is a monomorphism onto $G$ ( $i$ denotes the inclusion of $N$ into $M$.), and $H_{i}(X, A ; Z(G))=0$ for $j \leqq s$.

Lemma 2.5. If $N$ is an s-connected splitting, then $H_{s+1}(X, A ; Z(G))$ is $a$ finitely generated $Z(G)$ module.

Proof. By excision $H_{s+1}(X, A ; Z(G)) \cong H_{s+1}(B, N ; Z(G))$. Let $N_{s}$ denote the $s$ skeleton of $N$ (in some triangulation of $N$ ). Consider the homology exact sequence for the triple $\tilde{N}_{s} \subset \tilde{N} \subset \widetilde{B}$. We see that $H_{i}\left(B, N_{s} ; Z(G)\right)=H_{j}(B, N$; $Z(G))=0$ for $j \leqq s$, and that $H_{s+1}(B, N ; Z(G))$ is a quotient module of $H_{s+1}\left(B, N_{s} ; Z(G)\right)$. By Theorem $A$ of $[6]$ we have that $H_{s+1}\left(B, N_{s} ; Z(G)\right)$ is finitely generated, and hence $H_{s+1}(X, A ; Z(G))$ is finitely generated.

Consider $W=\overline{T(A)-A}$. This is a connected manifold with boundary where $\partial W=N \cup T(N)$. By considering the four spaces $T(N), B, W$, and $T^{-1}(B)$, we can show that the inclusion map of $T(N)$ into $W$ induces an isomorphism on fundamental groups. To do this, we use an argument analogous to the one used in showing that the inclusion of $N$ into $B$ induces an isomorphism on fundamental groups. Likewise, the inclusion of $N$ into $W$ induces an isomorphism on fundamental groups, and hence $H_{1}(W, N ; Z(G))=0$.

Next, consider the homology exact sequence for the triple $\tilde{A} \subset \widetilde{T(A)} \subset \tilde{X}$. In particular, consider the sequence

$$
H_{2}(X, A ; Z(G)) \stackrel{i *}{\rightarrow} H_{2}(X, T(A) ; Z(G)) \stackrel{\partial}{\rightarrow} H_{1}(T(A), A ; Z(G)) .
$$

By excision, $H_{1}(T(A), A ; Z(G)) \cong H_{1}(W, N ; Z(G))=0$, and hence $j_{*}$ is onto. The collection of modules $\left\{H_{i}\left(X, T^{m}(A) ; Z(G)\right)\right\}$, where $i$ is fixed, form a directed 
system whose maps are induced by the inclusions of $\left(\tilde{X}, \overparen{T^{m}(A)}\right)$ into $\left(\tilde{X}, \widetilde{\left.T^{n \prime}(A)\right)}\right.$ for $n^{\prime} \geqq m$. The direct limit of this system is $H_{i}(X, X ; Z(G))=0$. (See [3] Section 2.6.) For $i=2$, the two facts, $j_{*}$ onto and $H_{2}(X, A ; Z(G)$ ) finitely generated, imply that $H_{2}(X, A ; Z(G))=0$.

Hence, 2-connected splittings can always be found. Next, we show that $n-3$ connected splittings can always be found.

$\tilde{X}$ can also be considered as the universal covering space of $M$. As such, we can identify $\Pi_{1}(M)$ with the group of covering transformations of $\tilde{X}$. Let $t$ be an element of $\Pi_{1}(M)$ such that $f_{*}(t)$ is the generator of $\Pi_{1}\left(S^{1}\right)$ determined by the orientation of $S^{1}$ used in setting up the Pontryagin-Thom correspondence. Under our identification, $t: \tilde{X} \rightarrow \tilde{X}$ covers $T: X \rightarrow X$. $t$ is not uniquely defined, but for the remainder of this paper, our choice of $t$ will be fixed. Let $A$ be a subset of $X$ such that $A \subset T(A)$, then $t_{*}^{-1}$ induces an endomorphism of $H_{i}(\tilde{X}, \tilde{A})$, for each $i \cdot g \rightarrow t g t^{-1}$ is an automorphism of $G$ which induces an automorphism of $Z(G)$, and we denote this automorphism by $\alpha$. Then one sees easily that $t_{*}^{-1}$ is an $\alpha$ semi-linear endomorphism of $H_{i}(X, A ; Z(G))$, and hence this module together with this endomorphism can be considered as an object in $\mathfrak{C}^{*}(Z(G), \alpha)$. Let $B$ be a second subset of $X$ such that $B \subset T(B)$ and $A \subset B$. Then, $t_{*}^{-1}$ is an $\alpha$ semi-linear endomorphism of $H_{i}(B, A ; Z(G))$ and hence this pair defines an object in $\mathcal{C}^{*}(Z(G), \alpha)$. Consider the exact sequence of homology for the triple $\tilde{A} \subset \widetilde{B} \subset \tilde{X}$. By a straightforward verification, one shows that this is an exact sequence in the category $\mathcal{C}^{*}(Z(G), \alpha)$. If $C$ is a third subset of $X$ such that $C \subset T(C)$ and $B \subset C$, then the long exact sequence of homology for the triple $\tilde{A} \subset \widetilde{B} \subset \tilde{C}$ is an exact sequence in $\mathcal{C}^{*}(Z(G), \alpha)$.

Lemma 2.6. Suppose that $N$ is an $s-1$ connected splitting of $M$, then $t_{*}^{-1}$ is a nilpotent endomorphism of $H_{s}(X, A ; Z(G))$.

Proof. Let $j$ denote the inclusion map of $(\tilde{X}, \tilde{A})$ into $\left(\tilde{X}, T^{m}(A)\right)$. Using the two facts

$1^{\circ} H_{s}(X, A ; Z(G))$ is finitely generated, (Lemma 2.5)

$2^{\circ}$ direct $\operatorname{limit}_{r \varepsilon Z}\left\{H_{s}\left(X, T^{r}(A) ; Z(G)\right)\right\}$ equals 0 ,

we see that there exists an $m$ such that $j_{*}: H_{s}(X, A ; Z(G)) \rightarrow H_{s}\left(X, T^{m}(A)\right.$; $Z(G)$ ) equals 0. But $t^{-m}:(\tilde{X}, \tilde{A}) \rightarrow(\tilde{X}, \tilde{A})$ is the composite of $j:(\tilde{X}, \tilde{A}) \rightarrow$ $\left(\widetilde{X}, T^{m}(A)\right)$ and $t^{-m}:\left(\tilde{X}, T^{m}(A)\right) \rightarrow(\tilde{X}, \tilde{A})$, and hence $t_{*}^{-m}=\left(t_{*}^{-1}\right)^{m}=0$.

Lemma 2.7. There exist $n-3$ connected splittings.

Proof. Let $N$ be a $s-1$ connected splitting of $f$. (We know that 2 connected splittings always exist.) If $s \leqq n-3$, we will show how to construct a $s$-connected splitting by modifying $N$. An iteration of this process will finally produce an $n-3$ connected splitting of $f$. Let $\varphi$ denote $t_{*}^{-1}: H_{s}(X, A ; Z(G)) \rightarrow H_{s}(X, A$; $Z(G))$. By Lemma 2.6, $\varphi^{m}=0$ for some integer $m$. Let $K$ denote $H_{s}(X, A ; Z(G))$, and let $K_{i}=$ image $\varphi^{m-i}$. By Lemma $2.5, K$ is finitely generated, and hence 
$0=K_{0} \subset K_{1} \subset \cdots \subset K_{m}=K$ is a filtration of $K$ by finitely generated submodule such that $\varphi\left(K_{i}\right) \subset K_{i-1}$. Consider the homology exact sequence for the triple $\tilde{A} \subset \widetilde{T(A)} \subset \tilde{X}$. We see from it that $H_{i}(T(A), A ; Z(G))=0$ for $i<s-1$. By excision, this module is isomorphic to $\left(H_{i}(W, N ; Z(G))\right.$. By the theory of cobordism (see [8] Theorem 6.1.), $W=W_{1} \cup W_{2} \cup \ldots \cup W_{\text {, where }}$ each $W_{i}$ is an elementary cobordism (i.e., its image under the covering projection $\bar{p}: X \rightarrow M$ is an elementary cobordism) and $s-1 \leqq \operatorname{dim} W_{i} \leqq n-2$.

If $x \varepsilon H_{s}(W, N ; Z(G))$, then it is possible to arrange things so that $\operatorname{dim} W_{1}=s$ and so that there exists a generator $\bar{x}$ of $H_{s}\left(W_{1}, N ; Z(G)\right.$ such that $i_{*}^{\prime}(\bar{x})=x$ where $i^{\prime}$ denotes the inclusion map of $\left(\tilde{W}_{1}, \tilde{N}\right)$ into $(\tilde{W}, \tilde{N})$ (see [2], the proof of the Fundamental Lemma 4.8, for the details of this fact). Here we need that $s \leqq n-3$. Let $\hat{x}$ be one of a fixed finite collection of generators for $K_{1}$. By considering the exact homology sequence for the triple $\tilde{A} \subset \tilde{T}(A) \subset \tilde{X}$, we see that there exists an $x \varepsilon H_{\bullet}(T(A), A ; Z(G))$ such that $i_{*}(x)=\hat{x}$. This follows because $t^{-1}:(\tilde{X}, \tilde{A}) \rightarrow(\tilde{X}, \tilde{A})$ is the composite of the inclusion map $j:(\tilde{X}, \tilde{A}) \rightarrow$ $(\tilde{X}, T(A))$ and $t^{-1}:(\tilde{X}, T(A)) \rightarrow(\tilde{X}, \tilde{A})$. But, $\varphi(x) \varepsilon K_{0}=0$ and $t_{*}^{-1}: H_{s}(X$, $T(A) ; Z(G)) \rightarrow H_{\bullet}(X, A ; Z(G))$ is a monomorphism; hence, $j_{*}(\hat{x})=0$. Therefore,

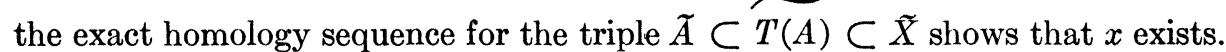
Pick $W_{1}$ as above. Then $\bar{p}\left(W_{1}\right)$ is an elementary cobordism between $N$ and a splitting $N^{\prime}$. Consider the homology sequence for the triple $\widetilde{A} \subset \widetilde{A}^{\prime} \subset \tilde{X}$ (where the particular lifting of $N^{\prime}$ to $X$ is determined by $\left.W_{1}\right)$. We see that $H_{i}\left(X, A^{\prime}\right.$; $Z(G))=0$ for $i<s$ and hence $N^{\prime}$ is also an $s-1$ connected splitting of $f$. Let $K^{\prime}$ denote $H_{s}\left(X, A^{\prime} ; Z(G)\right)$. Then, in dimension $s$ the sequence becomes: $Z(G) \stackrel{i^{*}}{\rightarrow} K \stackrel{i^{*}}{\rightarrow} K^{\prime} \rightarrow 0$. Let $\varphi^{\prime}$ denote $t_{*}^{-1}: K^{\prime} \rightarrow K^{\prime}$ and $K_{i}^{\prime}=$ image $\left(\varphi^{\prime}\right)^{m-1}$. Since $j_{*}$ is a map in $\mathcal{e}(Z(G), \alpha),\left(\varphi^{\prime}\right)^{m}=0$. Also, $j_{*}$ induces maps $K_{i} \rightarrow K_{i}^{\prime} \rightarrow 0$ for each $i$, and for $i=1$ this becomes $0 \rightarrow(\hat{x}) \rightarrow K_{1} \rightarrow K_{i}^{\prime} \rightarrow 0$. Hence, $K_{1}^{\prime}$ is generated by one fewer element than $K_{1}$, and therefore after repeating this process a finite number of times, we obtain an $s$ connected splitting. This completes the proof of Lemma 2.7.

Chapter III. The obstruction to psuedo fibering a circle. If $(N, \nu)$ is a splitting of $M$, then by $M_{N}$ we denote $M$ minus the interior of a tubular neighborhood of $N$.

Definition 3.1. $M$ pseudo fibers a circle with respect to $f \varepsilon H^{1}(M ; Z)$ if there exists a splitting $N$ of $M$ with respect to $f$ such that $M_{N}$ is an $h$-cobordism.

Remark. This definition is equivalent to the following condition. There exists a splitting $N$ such that the inclusion map $i: N \rightarrow X$ induces an isomorphism on fundamental groups and $H_{s}(X, A ; Z(G))=0$ for all $s$.

Definition 3.2. A splitting $(N, \nu)$ is called $s$-bi-connected if $(N, \nu)$ is $s$ connected and $(N,-\nu)$ is $(n-s)-1$ connected; that is, if $H_{2}(X, A ; Z(G))=0$ for $i \leqq s$ and $H_{i}(X, B ; Z(G))=0$ for $i \leqq(n-s)-1$. 
Note. If $(N, \nu)$ is a splitting with respect to $f \varepsilon H^{1}(M, Z)$, then $(N,-\nu)$ is a splitting with respect to $-f$.

Let $(N, \nu)$ be a splitting of $M$ with respect to $f$. Choose a lifting of $N$ to $X$ and denote this lifting also by $N$. Then, the cobordism $M_{N}$ can be identified with $\overline{T(A)-A}$, and hence we say that $M_{N}$ is a cobordism from $N$ to $T(N)$ and denote this by $\left(M_{N}, N, T(N)\right)$.

Lemma 3.3. A splitting $(N, \nu)$ is s-bi-connected if and only if there exists a handlebody decomposition of $\left(M_{N}, N, T(N)\right)$ consisting of only $s$ and $s+1$ dimensional handles.

Proof. If $(N, \nu)$ is s-bi-connected, then the inclusion maps of $N$ and $T(N)$ into $M_{N}$ induce isomorphisms on the fundamental groups. By considering the homology exact sequence for the triple $\widetilde{N} \subset \widetilde{M}_{N} \subset \widetilde{B}$, we see that $H_{i}\left(M_{N}, N\right.$; $Z(G))=0$ for $i<s$. Hence by [8] Theorem 6.1 , we see that $\left(M_{N}, N\right)$ has a handlebody decomposition consisting of handles only in dimensions $\geqq s$. By considering the exact sequence for $T(N) \subset M_{N} \subset T(A)$, we see that $H_{i}\left(M_{N}, T(N) ; Z(G)\right)=0$ for $i<(n-s)-1$. Hence by [8] Theorem 6.1, $\left(M_{N}, N, T(N)\right)$ has a handlebody decomposition consisting of only $s$ and $s+1$ dimensional handles.

The other half of Lemma 3.3 follows by considering the same homology exact sequences as in the first half, and using the fact that

$$
\text { direct } \operatorname{limit}_{s \varepsilon Z}\left\{H_{i}\left(X, T^{s}(A) ; Z(G)\right)\right\}=0,
$$

plus Lemma 2.5.

Lemma 3.4. There exist 2 bi-connected splittings.

Proof. This follows immediately from Lemma 2.7 applied to $-f$.

Lemma 3.5. If $(N, \nu)$ is an s-bi-connected splitting of $M$, then $H_{\bullet+1}(X, A$; $Z(G)$ ) is a projective $Z(G)$ module.

Proof. From Lemma 3.3, we see that $(B, N)$ is the homotopy type of a pair $(K, N)$, where $K$ is a C. W. complex obtained from $N$ by attaching $s$ and $s+1$ dimensional cells. Therefore, the chain groups for $(\tilde{K}, \tilde{N})$ vanish except in dimensions $s$ and $s+1$. Look at the following exact sequence:

$$
\begin{aligned}
0 \rightarrow & H_{s+1}(K, N ; Z(G)) \rightarrow C_{s+1}(K, N ; Z(G)) \\
& \stackrel{\partial}{\rightarrow} C_{s}(K, N ; Z(G)) \rightarrow H_{s}(K, N ; Z(G)) \rightarrow 0 .
\end{aligned}
$$

Using the facts that $H_{i}(K, N ; Z(G)) \cong H_{i}(X, A ; Z(G))$ and that $H_{s}(X, A$; $Z(G))=0$, the above sequence becomes:

$$
0 \rightarrow H_{s+1}(X, A ; Z(G)) \rightarrow C_{s+1}(K, N, Z(G)) \stackrel{\partial}{\rightarrow} C_{s}(K, N ; Z(G)) \rightarrow 0 .
$$

Since $C_{\mathrm{s}}(K, N ; Z(G))$ is a free $Z(G)$ module, this sequence splits. And since 
$C_{s+1}(K, N ; Z(G))$ is free, we see that $H_{s+1}(X, A ; Z(G))$ is a projective $Z(G)$ module.

By Lemma 2.5, we see that $H_{s+1}(X, A ; Z(G))$ is finitely generated, and by Lemma 2.6 we see that $t_{*}^{-1}$ is a nilpotent endomorphism of $H_{s+1}(X, A ; Z(G))$. Therefore, the pair $\left(H_{s+1}(X, A ; Z(G)), t_{*}^{-1}\right)$ is an object in the category $\mathfrak{e}(Z(G), \alpha)$. Denote this object by $c(N, \nu)$.

Remark. It appears that the isomorphism class of $c(N, \nu)$ depends on the particular lifting of $N$ to $X$. This is not the case. But we will not use this fact. Hence for our purposes, $c(N, \nu)$ depends on the lifting of $N$ to $X$. (See the first remark following the statement of Lemma 4.1.)

We will eventually define an obstruction $c(f) \varepsilon C(Z(G), \alpha)$ to psuedo fibering a circle by $c(f)=(-1)^{s+1} \sigma(c(N, \nu))$. But first we must show that this element is independent of the splitting $(N, \nu)$. To do this we assume that $X$ is the homotopy type of a finite C. W. complex. This is clearly a necessary condition which $M$ must satisfy in order to psuedo fiber a circle.

Remark. In the author's thesis, $c(f)$ was shown to be well defined under the weaker assumption that $X$ is dominated by a finite C. W. complex. The proof which follows uses results announced in [19].

Let $\varphi: X \rightarrow K$ be a homotopy equivalence, where $K$ is a finite C. W. complex. Let $T^{\prime}: K \rightarrow K$ be a cellular homotopy equivalence which makes the following diagram homotopy commutative:

$$
\begin{gathered}
\underset{\varphi \uparrow}{K} \stackrel{T^{\prime}}{\rightarrow} K \\
\underset{\uparrow^{\varphi}}{X} \\
X \stackrel{T}{\rightarrow} X .
\end{gathered}
$$

The mapping torus of $T, X_{r}$, is defined as $X \times[0,1] /(x, 0)=(T(x), 1)$. From the above diagram, we see that there exists a homotopy equivalence $F: X_{T} \rightarrow K_{T^{\prime}}$ such that $F^{-1}(K \times 0)=X \times 0$. If we consider the covering space $Z \rightarrow X \stackrel{\vec{P}}{\rightarrow} M$ as a principle $Z$ bundle, then $R \rightarrow X_{T} \stackrel{P^{\prime}}{\rightarrow} M$ is the associated principle $R$ bundle, where $p^{\prime}(x, t)=\bar{p}(x)$. Since $R$ is contractable, any two cross sections of $p^{\prime}$ are homotopic. Let $N$ be a splitting of $M$. Then we can construct a cross section $c_{N}$ of $p^{\prime}$ such that $c_{N}^{-1}(X \times 0)=N$. To do this, pick a function $g: M_{N} \rightarrow[0,1]$ such that $g^{-1}(0)=N$ and $g^{-1}(1)=T(N)$. Under the identification of $M_{N}$ with $\overline{T(A)-A}$, define $c_{N}(x)=(x, g(x))$. Consider $F_{N}=F \circ c_{N}: M \rightarrow K_{T^{\prime}}$, then $F_{N}$ is a homotopy equivalence between finite C. W. complexes. The remainder of Chapter III will be devoted to showing that $p\left(\tau\left(F_{N}\right)\right)=(-1)^{s+1} \sigma(c(N, \nu))$ if $N$ is $s$-bi-connected. Here, $\tau\left(F_{N}\right)$ denotes the Whitehead torsion of the homotopy equivalence $F_{N}$, and $p: \mathrm{Wh}\left(G \odot_{\alpha} Z\right) \rightarrow C(Z(G), \alpha)$ is the homomorphism defined in Chapter I. If $N^{\prime}$ is an $s^{\prime}$ bi-connected splitting then, since $c_{N^{\prime}}$ is homotopic to $c_{N}, \tau\left(F_{N^{\prime}}\right)=\tau\left(F_{N}\right)$. Hence $(-1)^{s+1} \sigma(c(N, \nu))=(-1)^{s^{\prime}+1} \sigma\left(N^{\prime}, \nu^{\prime}\right)$. 
Therefore, if we can establish the above formula then our obstruction $c(f)$ would be well defined.

Let $\mathbf{C}: C_{n} \rightarrow C_{n-1} \rightarrow \cdots \rightarrow C_{1} \rightarrow C_{0} \rightarrow 0$ be a based free chain complex over $R_{\alpha}[t]$, where each $C_{i}$ is finitely generated. Then, the basis for $\mathbf{C}$ induces a basis for $\mathrm{C}^{\prime}=\mathrm{C} \otimes_{R_{\alpha}[t]} R_{\alpha}\left[t, t^{-1}\right]$.

Lemma 3.6. Let $\mathbf{C}$ and $\mathbf{C}^{\prime}$ be given as above. Assume that $\mathbf{C}^{\prime}$ is acyclic, $H_{i}(\mathbf{C})=0$ for $i \neq s$, and Proj $\operatorname{dim}_{R_{\alpha}[t]} H_{s}(\mathbf{C}) \leqq 1$. Then, $\left(H_{s}(\mathbf{C}), t\right)$ is an object in $\mathrm{e}\left(R, \alpha^{-1}\right)$ and $p\left(\tau\left(\mathbf{C}^{\prime}\right)\right)=(-1)^{s} \sigma\left(H_{s}(\mathbf{C})\right.$, $\left.t\right)$, where $\tau\left(\mathbf{C}^{\prime}\right) \varepsilon K_{1}\left(R_{\alpha}\left[t, t^{-1}\right]\right)$ is the torsion of $\mathbf{C}^{\prime}$.

The proof of Lemma 3.6 will be postponed until the end of this chapter.

Let $\left(K, K_{1}, K_{2}\right)$ be a triad of finite C. W. complexes with $\Pi_{1}(K)=G \odot_{\alpha} Z$. Suppose that $\Pi_{1}\left(K_{2}\right)$ is mapped onto the normal subgroup $G$ of $\Pi_{1}(K)$ under the map induced from inclusion. Suppose that we can lift $K_{2}$ into the covering space $X$ of $K$ corresponding to $G$; such that $K_{2}$ divides $X$ into $A$ and $B$ with $T(A) \subset A$ where $T$ stands for the lifting of the element $t \varepsilon \Pi_{1}(K)$ to $X$. Assume that $K_{1}$ is a deformation retract of $K$. Let $Y$ denote the portion of $X$ covering $K_{1}$. Assume further that

(a) $H_{i}(A, A \cap Y ; Z(G))=0$ for $i \neq s$, and

(b) Proj $\operatorname{dim}_{Z(G) \alpha[t]} H_{s}(A, A \cap Y ; Z(G)) \leqq 1$.

Let $\tau\left(K, K_{1}\right) \varepsilon W h \Pi_{1}(K)=W h G \odot_{\alpha} Z$ denote the torsion of the pair $\left(K, K_{1}\right)$.

Lemma 3.7. $H_{s}(A, A \cap Y ; Z(G))$ is a finitely generated projective module over $Z(G)$. The covering transformation $t$ induces an $\alpha^{-1}$ semi-linear nilpotent endomorphism of $H_{s}(A, A \cap Y ; Z(G))$, and $p \tau\left(K, K_{1}\right)=(-1)^{s} \sigma\left(H_{s}(A, A \cap Y\right.$; $\left.Z(G)), t_{*}\right)$.

Proof. Let $\mathbf{C}=C_{*}(A, A \cap Y ; Z(G))$. Since $\mathbf{C}^{\prime} \cong C_{*}(X, Y ; Z(G)), \mathbf{C}$ satisfies the hypothesises of Lemma 3.6. Hence the conclusions of Lemma 3.7 follow directly from those for Lemma 3.6.

Now, let $f: K \rightarrow L$ be a homotopy equivalence of finite C. W. complexes. Suppose that $\Pi_{1}(L)=G \odot_{\alpha} Z$ and that $L_{1}$ is a subcomplex of $L$ with $\Pi_{1}\left(L_{1}\right)$ identified with $G$ under the map induced by inclusion. Let $X$ be the covering space of $L$ corresponding to $G$. Suppose that a lifting of $L_{1}$ into $X$ divides $X$ into $A_{L}$ and $B_{L}$ such that $T\left(A_{L}\right) \subset A_{L}$, where $T$ is the lifting of $t \varepsilon \Pi_{1}(L)$ to $X$. Let $Y$ be the corresponding covering space of $K ; f_{1}: Y \rightarrow X$ cover $f$ and $\tilde{Y}$ and $\tilde{X}$ denote the universal covering spaces of $Y$ and $X$ respectively. Let $\tilde{f}: \tilde{Y} \rightarrow \tilde{X}$ cover $f_{1}, K_{1}=f^{-1}\left(L_{1}\right), A_{K}=f_{1}^{-1}\left(A_{L}\right)$, and $B_{K}=f_{1}^{-1}\left(B_{L}\right)$. Assume that

(a) $\tilde{f}_{*}: H_{i}\left(A_{K} ; Z(G)\right) \rightarrow H_{i}\left(A_{L} ; Z(G)\right)$ is always epimorphic,

(b) $\tilde{f}_{*}$ is monomorphic except when $i=s$,

(c) Proj $\operatorname{dim}_{Z(G)_{\alpha}[t]} \operatorname{ker} \tilde{f}_{*} \leqq 1$,

(d) $f$ is cellular,

(e) $K, A_{K}$, and $B_{K}$ are subcomplexes of $Y$. 
Lemma 3.8. After making the above assumptions, ker $\tilde{f}_{*}$ is a finitely generated projective $Z(G)$ module. The covering transformation $t$ induces an $\alpha^{-1}$ semi-linear endomorphism on ker $\tilde{f}_{*}$, and

$$
p \tau(f)=(-1)^{s+1} \sigma\left(\operatorname{ker} \tilde{f}_{*}, t_{*}\right) .
$$

Proof. Form the mapping cylinder of $f$ and apply Lemma 3.7 to it.

Remark. Lemmas 3.6, 3.7, and 3.8 were originally announced in [19]. They represent joint work of the author and W. C. Hsiang.

Lemma 3.9. Let $(N, \nu)$ be an $s-1$ bi-connected splitting of $M$. Let $F_{N}: M \rightarrow K_{T}$, be the homotopy equivalence constructed in the paragraph preceding Lemma 3.6. Then $p\left(\tau\left(F_{N}\right)\right)=(-1)^{s} \sigma(C(N, \nu))$.

Proof. Let $X^{\prime}$ denote the $\infty$-cycle covering space of $K_{T^{\prime}}$. Then $K$ divides $X^{\prime}$ into two components which we denote by $A^{\prime}$ and $B^{\prime}$. There exists a lifting of $F_{N}, F_{N}^{\prime}: X \rightarrow X^{\prime}$, such that $\left(F_{N}^{\prime}\right)^{-1}\left(A^{\prime}\right)=A,\left(F_{N}^{\prime}\right)^{-1}\left(B^{\prime}\right)=B$, and $\left(F_{N}^{\prime}\right)^{-1}(K)=N$. Let $\widetilde{F}_{N}: \tilde{X} \rightarrow \widetilde{X}^{\prime}$ be a lifting of $F_{N}$ to the universal covering spaces. Consider the commutative diagram involving the two exact sequences in homology for $\tilde{A} \subset \tilde{X}$ and $\widetilde{A}^{\prime} \subset \tilde{X}^{\prime}$, and the map between them induced from $\widetilde{F}_{N}:(\tilde{X}, \widetilde{A}) \rightarrow$ $\left(\widetilde{X}^{\prime}, \widetilde{A}^{\prime}\right)$. It follows immediately that $\widetilde{F}_{N^{*}}: H_{i}(A ; Z(G)) \rightarrow H_{i}\left(A^{\prime} ; Z(G)\right)$ is an isomorphism for $i \neq s-1, s$. For $i=s-1$ and $s$, this diagram reduces to:

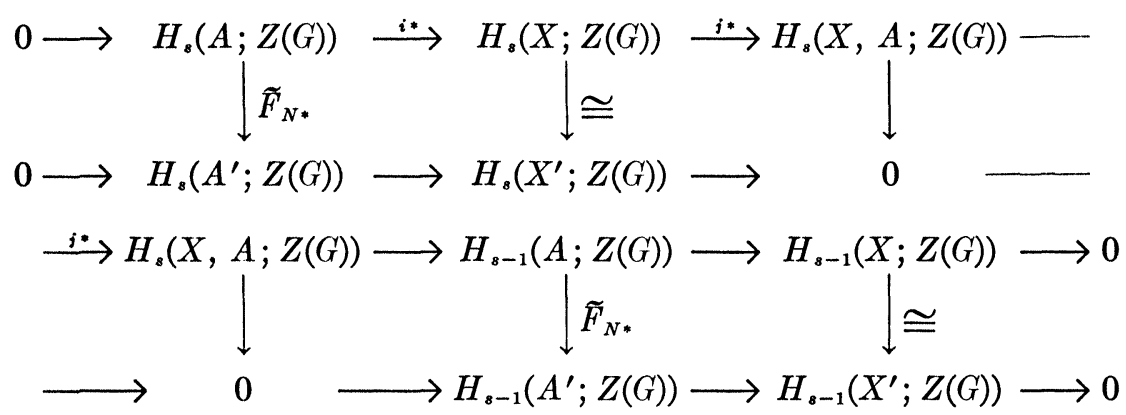

The maps in the above diagram are all $Z(G)_{\alpha}\left[t^{-1}\right]$ module maps. Next, we will show that $j_{*}$ is the zero map. By Lemma $2.5, H_{s}(X, A, Z(G))$ is a finitely generated $Z(G)$ module. Since direct $\operatorname{limit}_{i \varepsilon Z}\left\{H_{s}\left(X, T^{i}(A) ; Z(G)\right)\right\}=0$, there exists an integer $r$ such that $j_{*}^{\prime}: H_{s}(X, A ; Z(G)) \rightarrow H_{s}\left(X, T^{r}(A) ; Z(G)\right)$ is the zero map. This implies that $j_{*}^{\prime}: H_{s}\left(X, T^{-n}(A) ; Z(G)\right) \rightarrow H_{s}(X, A ; Z(G))$ is the zero map. But $j: \tilde{X} \rightarrow \underbrace{(\tilde{X}, \tilde{A})}$ is the composite of the inclusion map of $\tilde{X}$ into $\left(\tilde{X}, \overparen{\left.T^{-n}(A)\right)}\right.$ and $j^{\prime}:\left(\tilde{X}, T^{-n}(A)\right) \rightarrow(\tilde{X}, \tilde{A})$, and hence $j_{*}=0$. This implies that $i_{*}$ (see the above diagram) is an isomorphism. Therefore, $1^{\circ} F_{N^{*}}: H_{s}(A ; Z(G)) \rightarrow H_{s}\left(A^{\prime}\right.$; $Z(G))$ is an isomorphism, and $2^{\circ} \widetilde{F}_{N^{*}}: H_{s-1}(A ; Z(G)) \rightarrow H_{s-1}\left(A^{\prime} ; Z(G)\right)$ is an epimorphism with kernel isomorphic as a $Z(G)_{a}\left[t^{-1}\right]$ module to $H_{s}(X, A ; Z(G))$. Now Lemma 3.8 yields that $p\left(\tau\left(F_{N}\right)\right)=(-1)^{s} \sigma \subset(N, \nu)$, and this completes the proof of Lemma 3.9. 
Remark. If an $R_{\alpha}[t]$ module $X$ is projective as an $R$ module then the Proj $\operatorname{dim}_{R_{\alpha}[t]} X \leq 1$. (See [23] Lemma 9.) $H_{s}(X, A ; Z(G)$ ) is projective over $Z(G)$ by Lemma 3.5 , and hence Proj $\operatorname{dim}_{Z(G)_{\alpha}\left[t^{-1}\right]} H_{\star}(X, A ; Z(G)) \leqq 1$.

Definition 3.10. If $(N, \nu)$ is an $s$-bi-connected splitting of $M$ with respect to $f \varepsilon H^{1}(M, Z)$, then $c(f)=(-1)^{s+1} \sigma c(N, \nu)$.

Remark 1. $c(f)$ is independent of the choice of the splitting $(N, \nu)$. This follows from Lemma 3.9 together with the fact that if $\left(N^{\prime}, \nu^{\prime}\right)$ is a second splitting then $F_{N}$ is homotopic to $F_{N^{\prime}}$ and hence $\tau\left(F_{N}\right)=\tau\left(F_{N^{\prime}}\right)$.

Remark 2. If $M$ pseudo fibers a circle with respect to $f$, then $c(f)=0$. To see this, compute $c(f)$ using a splitting $N$ such that $\left(M_{N}, N\right)$ is an $h$-cobordism.

Remark 3. In Chapter V, we will see that $c(f)=0$ implies that $M$ pseudo fibers a circle with respect to $f$.

We conclude this chapter by giving the proof of Lemma 3.6. To do this, we need the following lemmas.

Let $\mathbf{C}: C_{n} \stackrel{\partial_{n}}{\rightarrow} C_{n-1} \rightarrow \cdots \rightarrow C_{1} \stackrel{\partial_{2}}{\rightarrow} C_{0} \rightarrow 0$ be a based finitely generated free acyclic chain complex over a ring $R$. Let $\zeta: C_{0} \rightarrow C_{1}$ be a splitting of $\partial_{1}$. Let

$$
\overline{\mathbf{C}}: \bar{C}_{n} \stackrel{\partial_{n}}{\rightarrow} \bar{C}_{n-1} \rightarrow \cdots \rightarrow \bar{C}_{0} \rightarrow 0
$$

be a new chain complex, where $\bar{C}_{i}=C_{i}$ if $i \neq 0$ and $2, \bar{C}_{2}=C_{2} \oplus C_{0}, \bar{C}_{0}=0$, $\bar{d}_{i}=d_{i}$ if $i \neq 1,2$ and $3, \bar{d}_{1}=0, \bar{d}_{2}(x, y)=d_{2}(x)+\zeta(y)$, and $\bar{d}_{3}(x)=\left(d_{3}(x), 0\right)$. Let $\mathbf{C}$ inherit a basis from $\mathbf{C}$ in the obvious way.

Lemma 3.11. If $\mathbf{C}$ and $\overline{\mathbf{C}}$ are as above, then $\tau(\mathbf{C})=\tau(\overline{\mathbf{C}})$.

Proof. See Barden [8].

Let $\zeta: C_{n-1} \rightarrow C_{n}$ be a splitting of $d_{n}$. Form a chain complex $\overline{\overline{\mathbf{C}}}$ as follows. Let $\overline{\bar{C}}_{i}=C_{i}$ if $i \neq n$ and $n-2, \overline{\bar{C}}_{n}=0, \overline{\bar{C}}_{n-2}=C_{n-2} \oplus C_{n}$. Let $\overline{\bar{d}}_{i}=d_{i}$ for $i \neq n, n-1$, and $n-2$. Let $\overline{\bar{d}}_{n}=0, d_{n-1}(x)=\left(d_{n-1}(x), \zeta(x)\right)$, and $\overline{\bar{d}}_{n-2}(x, y)=$ $d_{n-2}(x)$. Let $\overline{\overline{\mathbf{C}}}$ inherit a basis from $\mathbf{C}$ in the obvious way.

Lemma 3.12. $\tau(\mathbf{C})=\tau(\overline{\overline{\mathrm{C}}})$.

Proof. Similar to that of Lemma 3.11.

Lemma 3.13. Given the assumptions of Lemma 3.6. If $n>s+1$, then there exists a splitting $\zeta: C_{n-1} \rightarrow C_{n}$ of $d_{n}: C_{n} \rightarrow C_{n-1}$.

Proof. The sequence

*

$$
0 \rightarrow C_{n} \stackrel{\partial_{n}}{\rightarrow} C_{n-1} \rightarrow \cdots \rightarrow C_{s+1} \stackrel{\partial_{s+1}}{\rightarrow} B_{s} \rightarrow 0
$$

is exact. The sequence

$$
0 \rightarrow C_{s} / Z_{s} \stackrel{\partial_{s}}{\rightarrow} C_{s-1} \rightarrow \cdots \rightarrow C_{0} \rightarrow 0
$$


is also exact. From ${ }^{* *}$, we see that $C_{s} / Z_{s}$ is a projective $R_{\alpha}[t]$ module. From $0 \rightarrow Z_{s} \rightarrow C_{s} \rightarrow C_{s} / Z_{s} \rightarrow 0$, we see that $Z_{s}$ is projective. From $0 \rightarrow B_{s} \rightarrow Z_{s} \rightarrow$ $H_{s} \rightarrow 0$ and the fact that Proj $\operatorname{dim}_{R_{\alpha}[t]} H_{s} \leqq 1$, we see that $B_{s}$ is projective. Hence from *, we see that a splitting $\zeta$ of $d_{n}$ exists.

Proof of Lemma 3.6. If, in C, $C_{i}=0$ for $i \neq s$ and $s+1$, then lemma 3.6 would follow directly from the definition of $p: K_{1}\left(R_{\alpha}[t]\right) \rightarrow C(R, \alpha)$. Now by repeated use of Lemmas 3.11,3.12, and 3.13, we can reduce the proof of Lemma 3.6 to the above special case. That is, we can find a chain complex $\overline{\mathbf{C}}$ such that $\tau\left(\overline{\mathbf{C}} \otimes_{R_{\alpha}[t]} R_{\alpha}\left[t, t^{-1}\right]\right)=\tau\left(\mathbf{C} \otimes_{R_{\alpha}[t]} R_{\alpha}\left[t, t^{-1}\right]\right), \bar{C}_{i}=0$ for $i \neq s$ and $s+1$, and $H_{*}(\overline{\mathbf{C}}) \cong H_{*}(\mathbf{C})$. This completes the proof of Lemma 3.6.

Chapter IV. A duality formula for the obstruction to psuedo-fibering. If $(N, \nu)$ is an $s$-bi-connected splitting of $M$ with respect to $f \varepsilon H^{1}(M ; Z)$, then $(N,-\nu)$ is an $(n-s)-1$ bi-connected splitting of $M$ with respect to $-f . c(f)$ was defined in the preceding chapter to be $(-1)^{s+1} \sigma(c(N, \nu)) \varepsilon C(Z(G), \alpha)$. (Here $c(N, \nu)=\left(H_{s+1}(X, A ; Z(G)), t^{-1}\right) \varepsilon \mathfrak{e}(Z(G), \alpha)$.) Likewise, $c(-f)=(-1)^{n-s}$ $\sigma(c(N,-\nu)) \varepsilon C\left(Z(G), \alpha^{-1}\right)$. The purpose of this chapter is, by use of Poincare duality, to give a relationship between $c(f)$ and $c(-f)$. We will need, in fact, a slightly stronger result. Namely, we will establish a relationship between $c(N, \nu)$ as an object in $\mathcal{C}(Z(G), \alpha)$ and $c(N,-\nu)$ as an object in $\mathcal{C}\left(Z(G), \alpha^{-1}\right)$. The duality formula which we obtain is similar to that obtained by Stallings [20] for the torsion of a $h$-cobordism and Siebenmann [2] for the obstruction to adding a boundary to an open manifold.

We start this chapter by making some definitions of an algebraic nature. Let $\gamma$ be an anti-automorphism of a ring $R$ such that $\gamma \circ \gamma=i d$ and $\gamma \circ \alpha=$ $\alpha \circ \gamma$. We proceed to define a duality functor $\mathbb{D}: \mathcal{C}(R, \alpha) \rightarrow \mathfrak{C}\left(R, \alpha^{-1}\right)$. If $P \varepsilon \mathcal{\varepsilon}(R)$, we denote by $\overline{\operatorname{Hom}}_{R}(P, R)$ the collection of anti-homomorphisms of $P$ to $R$. ( $\varphi \varepsilon \overline{\operatorname{Hom}}_{R}(P, R)$ if $\varphi(x r)=\gamma(r) \varphi(x)$ for all $x \varepsilon P$ and $r \varepsilon R$, see [2], page 119.) Then $\overline{\mathrm{Hom}}_{R}(P, R) \varepsilon P(R)$. Let us denote this object by $D(P) . D$ is a contravariant exact functor from $\odot(R)$ to $\odot(R)$. Also, $D \circ D$ is naturally equivalent to the identity functor. Let $(P, f) \varepsilon \mathcal{e}(R, \alpha)$. If $\varphi \varepsilon D(P)$, we define $f^{*}(\varphi)=$ $\alpha^{-1} \circ \varphi \circ f$. Then $\left(D(P), f^{*}\right) \varepsilon \mathcal{C}\left(R, \alpha^{-1}\right)$. Denote this object by $\mathfrak{D}(P, f)$. This defines a contravariant exact functor from $\mathcal{C}(R, \alpha)$ to $\mathcal{C}\left(R, \alpha^{-1}\right)$. If $\mathscr{D}^{\prime}$ is the analogous functor from $\mathfrak{C}\left(R, \alpha^{-1}\right)$ to $\mathfrak{C}(R, \alpha)$, then $\mathscr{D}^{\prime} \circ \mathfrak{D}$ is naturally equivalent to the identity functor. Since $D$ takes free modules to free modules and is an exact functor, one easily sees that $D$ takes triangular objects to triangular objects.

Let $r$ be an invertible element in $R$. Let $\Omega_{r}$ denote right multiplication by $r$. Then $\mathcal{R}_{r}$ induces a functor which is an isomorphism between $\mathcal{e}(R, \alpha)$ and $\mathcal{C}\left(R, I_{r} \circ \alpha\right)$, where $I_{r}$ denotes the inner automorphism of $R$ which sends $s$ to $r^{-1} s r$. The functor $\mathcal{R}_{r}$ sends $(P, f)$ to $\left(P, \mathcal{R}_{r} \circ f\right)$.

If 1 denotes the identity element of $R$, then -1 denotes its additive inverse. Sometimes, we will be interested in the functor $\bar{D}=\mathbb{R}_{-1} \circ \mathbb{D}$ instead of $D$. $\bar{D}: \mathfrak{C}(R, \alpha) \rightarrow \mathfrak{C}\left(R, \alpha^{-1}\right)$ enjoys all of the properties which we listed above for $\mathfrak{D}$. 
Now, we return to our manifold $M$ in order to define a particular anti-automorphism of $Z(G)$.

Define $\theta: G \rightarrow Z_{2}$ (the cyclic group of order 2) as follows: if $g: \tilde{X} \rightarrow \tilde{X}$ is orientation preserving, let $\theta(g)=0$; if it is orientation reversing, then let $\theta(g)=1$. Define an anti-automorphism $\gamma$ of $Z(G)$ by $\gamma(g)=(-1)^{\theta(o)} g^{-1} \cdot \alpha(\gamma(g))=$ $(-1)^{\theta(\theta)} g^{-1} t^{-1}=(-1)^{\theta(t o t-1)}\left(t g t^{-1}\right)^{-1}=\gamma(\alpha(g))$. Therefore, $\gamma$ commutes with $\alpha$. Also $\gamma \circ \gamma=i d$.

Let $\triangle: \mathfrak{e}(Z(G), \alpha) \rightarrow \mathfrak{C}\left(Z(G), \alpha^{-1}\right)$ be the functor defined as follows:

$1^{\circ}$ if $t$ is orientation preserving, let $\triangle=\mathscr{D}$ (defined above).

$2^{\circ}$ if $t$ is orientation reversing, let $\triangle=\bar{D}$.

Lemma 4.1. If $(N, \nu)$ is an $s-1$ bi-connected splitting of $M$ with respect to $f$, then $\Delta c(N, \nu) \cong c(N,-\nu)$.

Remark. To be precise, Lemma 4.1 claims that given a lifting $\left(N_{1}, \nu_{1}\right)$ of $(N, \nu)$ to $X$ then there exists a second lifting $\left(N_{2}, \nu_{2}\right)$ such that $\triangle c\left(N_{1}, \nu_{1}\right) \cong$ $c\left(N_{2},-\nu_{2}\right)$. Our line of proof actually gives as a corollary that $c(N, \nu)$ is independent of liftings, i.e., $c\left(N_{1}, \nu_{1}\right) \cong c\left(N_{2}, \nu_{2}\right)$ for any pair of liftings $\left(N_{1}, \nu_{1}\right)$ and $\left(N_{2}, \nu_{2}\right)$. This follows because we show that for all sufficiently large positive integers $m, \triangle c\left(N_{1}, \nu_{1}\right) \cong c\left(T^{m} N_{1},-\nu_{1}\right)$ and hence $\triangle c\left(N_{1}, \nu_{1}\right) \cong \triangle c\left(N_{2}, \nu_{2}\right)$ which implies that $c\left(N_{1}, \nu_{1}\right) \cong c\left(N_{2}, \nu_{2}\right)$.

Remark. $\triangle$ induces a homomorphism from $C(Z(G), \alpha) \rightarrow C\left(Z(G), \alpha^{-1}\right)$ sending $x_{\varepsilon} C(Z(G), \alpha)$ to an element in $C\left(Z(G), \alpha^{-1}\right)$ which we denote by $\bar{x}$. Since $\triangle^{\prime} \circ \triangle$ is naturally equivalent to the identity functor, we see that $\bar{x}=x$.

Corollary 4.2. $\quad c(-f)=(-1)^{n+1} \overline{c(f)}$.

The remainder of this chapter will be devoted to proving Lemma 4.1.

Let $c(N, \nu)=(P, \varphi)$ and $c(N,-\nu)=(Q, \psi)$. Then there exists an integer $m$ such that $\varphi^{m}=\psi^{m}=0$. Consider the homology exact sequence for the triple $\tilde{A} \subset T^{m}(A) \subset \tilde{X}$. Since $\varphi^{m}=0$, we see that $j_{*}=0$ where $j:(X, A) \rightarrow\left(X, T^{m}(A)\right)$, Therefore $(P, \varphi) \cong\left(H_{s}\left(T^{m}(A), A ; Z(G)\right), t_{*}^{-1}\right)$. Likewise $(Q, \psi) \cong\left(H_{n-s}(B\right.$. $\left.\left.T^{m}(B) ; Z(G)\right), t_{*}\right)$. Let $W=\overline{T_{m}(A)-A}$.

Let $u$ represent the fundamental homology class (perhaps represented by an infinite chain) for $(\tilde{W}, \partial \tilde{W})$. (This amounts to choosing an orientation for $\tilde{W}$.) Poincare duality implies that $\wedge_{u}: H_{\text {comp }}^{s}(\tilde{W}, \tilde{N} ; Z) \rightarrow H_{n-s}\left(\tilde{W}, T^{m}(N) ; Z\right)$ is an isomorphism as $Z$ modules (see [9], page 225). By $H_{\text {comp }}^{*}$ we denote cohomology with compact supports.

Recall the following result about cap products. Let $\left(\mathrm{X}, A_{1}, A_{2}\right)$ and $\left(Y, B_{1}, B_{2}\right)$ be triads, where the $A_{i}$ are closed subsets of $\mathrm{X}$ and the $B_{i}$ are closed subsets of $Y$. Let $f:\left(X, A_{1}, A_{2}\right) \rightarrow\left(Y, B_{1}, B_{2}\right)$ be a proper map; $f_{i}, i=1$ and 2, denote $f_{i}:\left(X, A_{i}\right) \rightarrow\left(Y, B_{i}\right)$, and $\bar{f}$ denote $f:\left(X, A_{1} \cup A_{2}\right) \rightarrow\left(Y, B_{1} \cup B_{2}\right)$. Let $u \varepsilon H_{\text {oomp }}^{a}\left(Y, B_{1}\right)$, and $z \varepsilon H_{n}^{\text {inf }}\left(X, A_{1} \cup A_{2}\right)$. ( $H_{*}^{\text {inf }}$ denotes homology with infinite chains.) 
Lemma 4.3. $\quad f_{2_{*}}\left(f_{1}^{*}(u)^{\cap} z\right)=u^{\cap} f_{*}(z)$.

If $g \varepsilon G$, then $g:\left(\tilde{W}, \tilde{N}, T^{m}(N)\right) \rightarrow\left(\tilde{W}, \tilde{N}, T^{m}(N)\right)$. Applying Lemma 4.3 to $g$, we obtain the following commutative diagram:

$$
\begin{aligned}
& H_{\text {comp }}^{s}(\tilde{W}, \tilde{N} ; Z) \stackrel{\cap_{u}}{\longrightarrow} H_{n-s}(\tilde{W}, \underbrace{T^{m}(N)}_{\left.\downarrow^{(1)}\right)^{*}} ; Z) \\
& H_{\text {comp }}^{s}(\tilde{W}, \tilde{N} ; Z) \stackrel{\cap^{* *(u)}}{\longrightarrow} H_{n-s}\left(\tilde{W}, T_{T^{m}(N)} ; Z\right) .
\end{aligned}
$$

If $g$ is orientation preserving, then $g_{*}(u)=u$, while if $g$ reverses orientation, then $g_{*}(u)=-u$. Hence, if we change $H_{\text {comp }}^{s}(\tilde{W}, \tilde{N} ; Z)$ into a right $Z(G)$ by use of an anti-automorphism $\gamma$, then $\wedge_{u}$ becomes an isomorphism between right $Z(G)$ modules.

Next, we will show a stronger result, namely,

$$
\left(H_{\text {comp }}^{:}(\tilde{W}, \tilde{N} ; Z), \pm t^{-1 *}\right) \cong c(N,-\nu)
$$

as objects in $\mathcal{e}\left(Z(G), \alpha^{-1}\right)$.

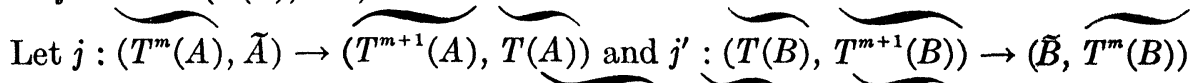
denote inclusion maps. Denote $t^{-1}:\left(T^{m+1}(A), T(A)\right) \rightarrow\left(T^{m}(A), \widetilde{A}\right)$ by $F$ and $t:\left(\widetilde{B}, T_{\left.T^{m}(B)\right)}^{\rightarrow}\left(T(B), T^{m+1}(B)\right)\right.$ by $F^{\prime}$. Then $\left.\left.t^{-1}: \overparen{\left(T^{m}(A)\right.}, \tilde{A}\right) \rightarrow \overparen{\left(T^{m}(A)\right.}, \tilde{A}\right)$ is the composite of $j$ and $F$. Likewise, $t:\left(\widetilde{B}, \widetilde{\left.T^{m}(B)\right)} \rightarrow\left(\widetilde{B}, \widetilde{T^{m}(B)}\right)\right.$ is the composite of $F^{\prime}$ and $j^{\prime}$.

By several applications of Lemma 4.3, we obtain the following commutative diagram:

$$
\begin{aligned}
& \left.H_{n-s}\left(\widetilde{B}, \overparen{T^{m}(B)} ; Z\right) \stackrel{{ }^{\prime *}}{\longrightarrow} H_{n-s} \overparen{(T(B)}, \overparen{T^{m+1}(B)} ; Z\right) \\
& H_{\text {comp }}^{s} \overbrace{\left(T^{m}(A)\right.}^{\uparrow}, \tilde{A} ; Z) \stackrel{i^{*}}{\longrightarrow} H_{\text {comp }}^{s}\left(T_{T^{m+1}(A)}^{\uparrow \cap_{u^{\prime}}}, \widetilde{T(A)} ; Z\right) .
\end{aligned}
$$

In this diagram $u^{\prime}$ represents the fundamental homology class of $\overline{T^{m+1}(A)-T(A)}$ (since $\widetilde{X}$ is an orientable manifold, we can pick the fundamental class $u^{\prime}$ to be consistent with the class $u$ ).

Using Lemma 4.3 again, we obtain that the diagram

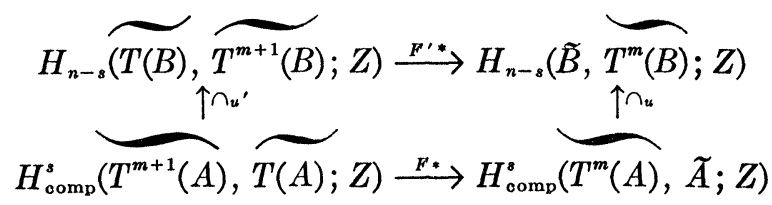

either commutes or skew commutes, depending on whether $t$ is orientation preserving or reversing. Putting diagrams * and ** together, we obtain the diagram 


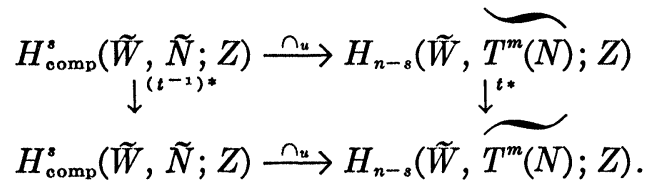

This diagram commutes if $t$ is orientation preserving and skew commutes if $t$ reverses orientation.

Hence we have completed step $1^{\circ}$ in the proof of Lemma 4.1.

Step $1^{\circ} . \quad\left(H_{\text {comp }}^{s}(\tilde{W}, \tilde{N} ; Z), \pm t^{-1 *}\right) \cong c\left(T^{m}(N),-\nu\right)$ as objects in $\mathrm{e}\left(Z(G), \alpha^{-1}\right)$. Notice that $+t^{-1 *}$ appears if $t$ is orientation preserving. Otherwise, $-t^{-1 *}$ appears.

If $R$ is a ring and $B$ is an automorphism of $R$, we denote by $\mathcal{C}^{* *}(R, \Theta)$ the category whose objects are pairs $(K, \zeta)$, where $K$ is a left $R$ module and $\zeta$ is a $B$ semi-linear endomorphism of $K$. Using this definition, step $2^{\circ}$ in the proof of Lemma 4.1 has the following formulation.

Step $\mathscr{Z}^{\circ} . \quad\left(H_{\text {comp }}^{s}(\tilde{W}, \tilde{N} ; Z), t^{-1 *}\right) \cong\left(\operatorname{Hom}_{Z(G)}(P, Z(G)), \operatorname{Hom}\left(\varphi, \alpha^{-1}\right)\right)$ as objects in $\mathfrak{C}^{* *}\left(Z(G), \alpha^{-1}\right)$. If we change both of these objects from $\mathfrak{C}^{* *}(Z(G)$, $\left.\alpha^{-1}\right)$ into objects in $\mathcal{C}\left(Z(G), \alpha^{-1}\right)$ by use of the anti-automorphism $\gamma$ and put steps $1^{\circ}$ and $2^{\circ}$ together, we obtain that $c\left(T^{m}(N),-\nu\right) \cong \Delta c(N, \nu)$. This proves Lemma 4.1, modulo the demonstration of step $2^{\circ}$.

Demonstration of step $2^{\circ}$. Since $(W, N)$ has a handlebody decomposition consisting of only $s$ and $s-1$ dimensional handles, $(P, \varphi)$ is isomorphic to the kernel of $\partial$ in the free based $Z(G)$ chain complex thus determined:

$$
\left(C_{s}, \varphi_{s}\right) \stackrel{\partial}{\rightarrow}\left(C_{s-1}, \varphi_{s-1}\right) \text {. }
$$

Here, both $C_{s}$ and $C_{s-1}$ are finitely generated and $\partial$ is a map in $\mathrm{e}^{*}(Z(G), \alpha)$. The cokernel of $\partial$ is isomorphic to $H_{s}\left(\tilde{X}, T^{m}(A)\right.$ ). (Look again at the homology sequence for the triple $\tilde{A} \subset T^{m}(A) \subset \tilde{X}$ and use the fact that $j_{*}=0$.) But $H_{s}\left(\tilde{X}, T^{m}(A)\right)$ is projective, and hence ker $\partial$ is a direct summand of $C_{.}$. Look at the chain complex

* $\quad\left(\operatorname{Hom}_{Z(G)}\left(C_{\&}, Z(G)\right), \operatorname{Hom}\left(\varphi_{s}, \alpha^{-1}\right)\right)$

$$
\stackrel{\text { Hom }(\partial, i d)}{\longrightarrow}\left(\operatorname{Hom}_{Z(G)}\left(C_{s-1}, Z(G)\right) \text {, Hom }\left(\varphi_{s-1}, \alpha^{-1}\right)\right) \text {. }
$$

The cokernel of Hom $(\partial, i d)$ is isomorphic to

$$
\left(\operatorname{Hom}_{Z(G)}(P, Z(G)), \quad \operatorname{Hom}\left(\varphi, \alpha^{-1}\right)\right) .
$$

But $\left(H_{\text {oomp }}^{s}(\tilde{W}, \tilde{N} ; Z), t^{-1 *}\right)$ is isomorphic to the cokernel of Hom $(\partial, i d)$

** $\quad\left(\operatorname{Hom}_{Z}^{\text {comp }}\left(C_{s}, Z\right), \operatorname{Hom}\left(\varphi_{s}, i d\right)\right)$

$$
\stackrel{\text { Hom }(\partial, i d)}{\longrightarrow}\left(\operatorname{Hom}_{Z}^{\text {eomp }}\left(C_{s-1}, Z\right), \operatorname{Hom}\left(\varphi_{s-1}, i d\right)\right),
$$


where $\operatorname{Hom}_{Z}^{\text {comp }}\left(C_{s}, Z\right)$ denotes the $Z$ homomorphism with vanish on all but a finite number of the distinguished basis elements of $C_{s}$. (The distinguished $Z$ basis for $C_{s}$ is determined by the distinguished $Z(G)$ basis for $C_{s}$ by the action of $G$.) But the complexes * and ${ }^{* *}$ are isomorphic in $\mathfrak{C}^{* *}(Z(G), \alpha)$ (see [9], page 223). An explicit isomorphism is the following. Let $h: Z(G) \rightarrow Z$ be the $Z$ linear map determined by $h(g)=0$ if $g \varepsilon G, g \neq 1$, and $h(1)=1$. Then the map of $\operatorname{Hom}_{Z(G)}\left(C_{i}, Z(G)\right)$ into $\operatorname{Hom}_{Z}^{\text {comp }}\left(C_{i}, Z\right)(i=s$ or $s-1)$ given by sending $y$ to $h \circ y$ is the isomorphism.

This completes the demonstration of step $2^{\circ}$, and hence Lemma 4.1 is proven.

Chapter V. M pseudo fibers a circle if an only if $c(f)=\mathbf{0}$. In this chapter, we show that if $c(f)=0$, then $M$ pseudo fibers a circle with respect to $f$. This will be a consequence of the following more general result.

Lemma 5.1. Let $(N, \nu)$ be an $s$ bi-connected splitting of $M$ where $2 \leqq s \leqq n-4$, and let $(P, \varphi)$ be an object from $\mathrm{e}(Z(G), \alpha)$ such that $\sigma(P, \varphi)=\sigma(c(N, \nu))$. Then, there exists an $s$ bi-connected splitting $\left(N^{\prime}, \nu^{\prime}\right)$ such that $c\left(N^{\prime}, \nu^{\prime}\right) \cong(P, \varphi)$.

Suppose that $c(f)=0$. Then, there exists a 2 bi-connected splitting $(N, \nu)$ such that $\sigma(c(N, \nu))$ is the identity element of $C(Z(G), \alpha)$. But $\sigma(0, o)$ is also the identity element of $C(Z(G), \alpha)$. Hence, by Lemma 5.1 , there exists a 2 biconnected splitting $\left(N^{\prime}, \nu^{\prime}\right)$ of $M$ with respect to $f$, such that $H_{3}\left(X, A^{\prime} ; Z(G)\right)=0$. By the remark following Definition 3.1, this shows that $M$ pseudo fibers a circle with respect to $f$. This fact together with Remark $2^{\circ}$ following Definition 3.10 proves the following theorem.

Theorem 2.5. $M$ pseudo fibers a circle with respect to $f$ if and only if $c(f)=0$.

The remainder of this chapter will be devoted to the proof of Lemma 5.1.

Lemma 5.3. Let $(N, \nu)$ be an $s$ bi-connected splitting of $M$, where $2 \leqq s \leqq$ $n-4$, and let $\left.0 \rightarrow\left(P_{2}, \varphi_{2}\right) \rightarrow\left(P_{1}, \varphi_{1}\right) \rightarrow P_{0}, \varphi_{0}\right) \rightarrow 0$ be an exact sequence in $\mathrm{e}(Z(G), \alpha)$. Suppose that $\left(P_{1}, \varphi_{1}\right)$ is a triangular object in $\mathrm{e}(Z(G), \alpha)$ and that $c(N, \nu) \cong\left(P_{0}, \varphi_{0}\right)$. Then, there exists an $s+1$ bi-connected splitting $\left(N^{\prime}, \nu^{\prime}\right)$ such that $c\left(N^{\prime}, \nu^{\prime}\right) \cong\left(P_{2}, \varphi_{2}\right)$.

The proof of Lemma 5.3 will be given at the end of this chapter.

Next, we need the following algebraic lemma.

Lemma 5.4. Let $0 \rightarrow P_{2} \stackrel{i}{\rightarrow} P_{1} \stackrel{p}{\rightarrow} P_{0} \rightarrow$ and $0 \rightarrow Q_{2} \stackrel{i^{\prime}}{\rightarrow} Q_{1} \stackrel{p^{\prime}}{\rightarrow} Q_{0} \rightarrow 0$ be two exact sequences in $\odot(R)$, and let $y_{i}: Q_{i} \rightarrow P_{i}, i \neq 1$, be $\alpha$ semi-linear homomorphisms. Then, there exists an $\alpha$ semi-linear homomorphism $y_{1}: Q_{1} \rightarrow P_{1}$ such that $y_{1} \circ i^{\prime}=i \circ y_{2}$ and $p \circ y_{1}=y_{0} \circ p^{\prime}$.

Proof. Let $c$ and $c^{\prime}$ be splittings of $p$ and $p^{\prime}$ respectively. Then, $P_{1}=$ image $i \oplus$ image $c$ and $Q_{1}=$ image $i^{\prime} \oplus$ image $c^{\prime}$. With these identifications $y_{0}$ and $y_{2}$ clearly define $y_{1}$.

Proof of Lemma 5.1. Let $(N, \nu)$ be an $s$ bi-connected splitting of $M$, and let $(P, \varphi)$ be an object from $\mathfrak{e}(Z(G), \alpha)$ such that $\sigma(P, \varphi)=\sigma(c(N, \nu))$. From 
the explicit construction of $\mathrm{e}(Z(G), \alpha)$ given in Chapter I, we see that the isomorphism class of $(P, \varphi)$ is related to the isomorphism class of $c(N, \nu)$ by a sequence of elementary equivalences of type $1^{\prime \circ}$ or $2^{\prime \circ}$. Hence, to prove Lemma 5.1 , it suffices to prove it in the special case when $(P, \varphi)$ is related to $c(N, \nu)$ by an elementary equivalence of type $1^{\prime \circ}$ or $2^{\prime \circ}$. Let us recall the statement of relations $1^{\prime \circ}$ and $2^{\prime \circ}$.

$1^{\prime \circ}$ If $F$ is free, then $(P, f) \sim(P \oplus F, f \oplus 0)$.

$2^{\prime \circ}$ If $\left(P_{2}, f_{2}\right) \rightarrow\left(P_{1}, f_{1}\right) \rightarrow\left(P_{0}, f_{0}\right) \rightarrow 0$ is exact in $\mathcal{C}(R, \alpha)$, then $\left(P_{1}, f_{1}\right) \sim$ $\left(P_{2} \oplus P_{0}, f_{2} \oplus f_{0}\right)$.

First, let us consider relations $1^{\prime \circ}$. Let $0 \rightarrow\left(P_{2}, \varphi_{2}\right) \rightarrow\left(P_{1}, \varphi_{1}\right) \rightarrow\left(P_{0}, \varphi_{0}\right) \rightarrow 0$ be an exact sequence in $\mathcal{C}(Z(G), \alpha)$. Suppose that $\left(P_{1}, \varphi_{1}\right)$ is triangular and that $c(N, \nu) \cong\left(P_{0}, \varphi_{0}\right)$. Then by Lemma 5.3 there exists an $s+1$ bi-connected splitting $\left(N_{1}, \nu_{1}\right)$ of $M$ such that $c\left(N_{1}, \nu_{1}\right) \cong\left(P_{2}, \varphi_{2}\right)$. By Lemma $4.1, c\left(N_{1},-\right.$ $\left.\nu_{1}\right) \cong \triangle\left(P_{2}, \varphi_{2}\right)$. But $\triangle$ is an exact contravariant functor, and hence $0 \leftarrow$ $\triangle\left(P_{2}, \varphi_{2}\right) \leftarrow \Delta\left(P_{1}, \varphi_{1}\right) \leftarrow \Delta\left(P_{0}, \varphi_{0}\right) \leftarrow 0$ is exact in $\mathcal{C}\left(Z(G), \alpha^{-1}\right)$. Also as remarked in Chapter IV, $\triangle\left(P_{1}, \varphi_{1}\right)$ is triangular. Let $F$ be a finitely generated free $Z(G)$ module, and let $g: \Delta\left(P_{0}, \varphi_{0}\right) \rightarrow \triangle\left(P_{1}, \varphi_{1}\right)$ denote the map in the exact sequence above. Then $0 \rightarrow \triangle\left(P_{0}, \varphi\right) \oplus(F, 0) \stackrel{{ }^{\oplus i d}}{\rightarrow} \Delta\left(P_{1}, \varphi_{1}\right) \oplus(F, 0)$ $\rightarrow \triangle\left(P_{2}, \varphi_{2}\right) \rightarrow 0$ is exact, and $\triangle\left(P_{1}, \varphi_{1}\right) \oplus(F, 0)$ is triangular. Hence by Lemma 5.3 there exists a splitting $\left(N_{2}, \nu_{2}\right)$ with respect to $-f$ such that $c\left(N_{2}, \nu_{2}\right)$ $\cong \triangle\left(P_{0}, \varphi_{0}\right) \oplus(F, 0)$. By Lemma 4.1 again, $c\left(N_{2},-\nu_{2}\right) \cong \triangle^{\prime} \triangle\left(P_{0}, \varphi_{0}\right)$ $\oplus \triangle^{\prime}(F, 0)$. But $\triangle^{\prime} \circ \triangle$ is naturally equivalent to the identity functor and $\triangle^{\prime}(F) \cong F$. Hence $c\left(N_{2},-\nu_{2}\right) \cong\left(P_{0}, \varphi\right) \oplus(F, 0)$, and thus relation $1^{\prime \circ}$ going in one direction can be realized. The opposite direction can be realized in a similar fashion.

Next we consider relation $2^{\prime \circ}$. Let

$$
0 \rightarrow\left(P_{2}, \varphi_{2}\right) \rightarrow\left(P_{1}, \varphi_{1}\right) \rightarrow\left(P_{0}, \varphi_{0}\right) \rightarrow 0
$$

and

$$
0 \rightarrow\left(Q_{2}, \psi_{2}\right) \rightarrow\left(Q_{1}, \psi_{1}\right) \rightarrow\left(Q_{0}, \psi_{0}\right) \rightarrow 0
$$

be two exact sequences in $\mathcal{e}(Z(G), \alpha)$, and let $y_{0}, y_{0}^{\prime}: Q_{0} \rightarrow P_{0}$ be two $\alpha$ semilinear maps. Define $g_{0}: P_{0} \oplus Q_{0} \rightarrow P_{0} \oplus Q_{0}$ by $g_{0}(a, b)=\left(\varphi_{0}(a) \oplus y_{0}(b), \psi_{0}(b)\right)$, and $g_{0}^{\prime}: P_{0} \oplus Q_{0} \rightarrow P_{0} \oplus Q_{0}$ by $g_{0}^{\prime}(a, b)=\left(\varphi_{0}(a) \oplus y_{0}^{\prime}(b), \psi_{0}(b)\right)$. Then applying Lemma 5.4 twice with $y_{2}=0=y_{2}^{\prime}$, we obtain the following two exact sequences in $\mathfrak{e}(Z(G), \alpha)$ :

$$
0 \rightarrow\left(P_{2} \oplus Q_{2}, \varphi_{2} \oplus \psi_{2}\right) \rightarrow\left(P_{1} \oplus Q_{1}, g_{1}\right) \rightarrow\left(P_{0} \oplus Q_{0}, g_{0}\right) \rightarrow 0
$$

and

$$
0 \rightarrow\left(P_{2} \oplus Q_{2}, \varphi_{2} \oplus \psi_{2}\right) \rightarrow\left(P_{1} \oplus Q_{1}, g_{1}^{\prime}\right) \rightarrow\left(P_{0} \oplus Q_{0}, g_{0}^{\prime}\right) \rightarrow 0 .
$$

Assume that $\left(P_{1}, \varphi_{1}\right)$ and $\left(Q_{1}, \psi_{1}\right)$ are triangular. Then $\left(P_{1} \oplus Q_{1}, g_{1}\right)$ and $\left(P_{1} \oplus Q_{1}, g_{1}^{\prime}\right)$ are triangular. Suppose that $c(N, \nu) \cong\left(P_{0} \oplus Q_{0}, g_{0}\right)$. Then by Lemma 5.3 there exists an $s+1$ bi-connected splitting $\left(N_{1}, \nu_{1}\right)$ such that $c\left(N_{1}, \nu_{1}\right) \cong\left(P_{2} \oplus Q_{2}, \varphi_{2} \oplus \psi_{2}\right)$. By Lemma $4.1 c\left(N_{1},-\nu_{1}\right) \cong \triangle\left(P_{2} \oplus Q_{2}\right.$, 
$\left.\varphi_{2} \oplus \psi_{2}\right)$. But since $\triangle$ is contravariant and exact, $0 \leftarrow \triangle\left(P_{2} \oplus Q_{2}, \varphi_{2} \oplus \psi_{2}\right) \leftarrow$ $\triangle\left(P_{1} \oplus Q_{1}, g_{1}^{\prime}\right) \leftarrow \triangle\left(P_{0} \oplus Q_{0}, g_{0}^{\prime}\right) \leftarrow 0$ is exact in $\mathcal{C}\left(Z(G), \alpha^{-1}\right)$. By Lemma 5.3, there exists an $n-(s+1)$ bi-connected splitting $\left(N_{2}, \nu_{2}\right)$ of $M$ with respect to $-f$ such that $c\left(N_{2}, \nu_{2}\right) \cong \triangle\left(P_{0} \oplus Q_{0}, g_{0}^{\prime}\right)$. Applying Lemma 4.1 again, we see that $\left(N_{2},-\nu_{2}\right)$ is an $s$ bi-connected splitting of $M$ with respect to $f$ such that $c\left(N_{2},-\nu_{2}\right)=\left(P_{0} \oplus Q_{0}, g_{0}^{\prime}\right)$, and hence relation $2^{\prime \circ}$ can be realized. This completes the proof of Lemma 5.1, modulo the proof of Lemma 5.3.

Proof of Lemma 5.3. Let $p: P_{1} \rightarrow H_{s+1}(X, A ; Z(G))$ denote the map from $P_{1}$ to $P_{0}$ composed with the given isomorphism from $P_{0}$ to $H_{s+1}(X, A ; Z(G))$. Since $\left(P_{1}, \varphi_{1}\right)$ is triangular, there exists a filtration $0=F_{0} \subset F_{1} \subset \cdots \subset$ $F_{m}=P_{1}$, such that $\varphi_{1}\left(F_{i+1}\right) \subset F_{i}$, where each $F_{i}$ is free, and $Q_{i}=F_{i} / F_{i-1}$ is free on one generator.

We prove Lemma 5.3 by induction, where the i-th inductive statement is the following.

Inductive statement. After exchanging $i(s+1)$ dimensional handles from $B$ to $A$, we can find a monomorphism $\rho: H_{s+1}(R, N, Z(G)) \rightarrow P_{1}$, where $R$ is the cobordism formed from the union of the $i$ exchanged elementary cobordisms. The image of $\rho$ is $F_{i} ; \rho$ is a map in $\mathcal{C}(Z(G), \alpha)$; and $p \circ \rho=i_{*}$, where $i$ is the inclusion map of $(R, N)$ into $(X, A)$.

Remark. By exchanging $i(s+1)$ dimensional handles from $B$ to $A$, we mean lifting a sequence of $(s+1)$ dimensional elementary cobordisms (see Definition 2.2.) to $X$, so that the lifted sequence starts at the given lifting of $(N, \nu)$ to $X$.

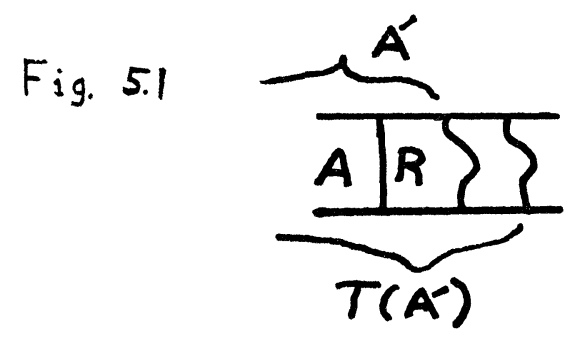

We show next how Lemma 5.3 follows from the $m$-th inductive statement, and then we will conclude this chapter by proving the inductive statement.

Consider the inductive statement for $i=m$, and the homology exact sequence for the triple $A \subset A^{\prime} \subset X$. We obtain the following diagram, $A^{\prime}=A \cup R$ :

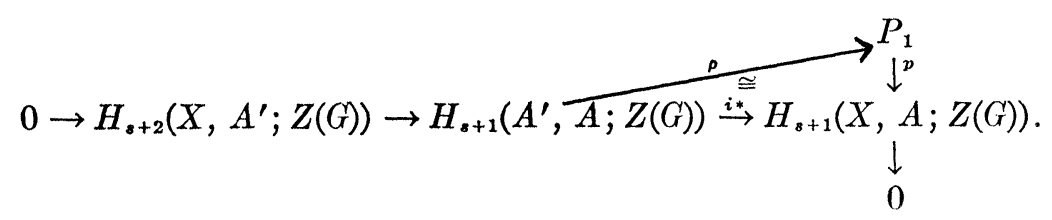


Since all of the maps in this diagram are maps in $\mathfrak{e}(Z(G), \alpha)$, we see that $\left(H_{s+2}\left(X, A^{\prime} ; Z(G)\right), t^{-1 *}\right) \cong\left(P_{2}, \varphi_{2}\right)$. One also shows easily that the new splitting is $s+1$ bi-connected, and hence we have proven Lemma 5.3, modulo the proof of the inductive statement.

Proof of the inductive statement. The 0-th statement is clearly true. Let us assume the truth of the $i$-th statement and try to prove the $(i+1)$-st statement. Pick $e \varepsilon F_{i+1}$ such that $F_{i} \oplus\{e\}=F_{i+1}$. Let $b=p(e)$. Denote the region obtained by exchanging the first $i$ handles by $R$, and let $A^{\prime}=A \cup R$. Since $\varphi_{1}(e) \varepsilon F_{i}, \varphi_{1}(e)$ is in the image of $\rho$. Let $c$ be that element of $H_{s+1}(R, N)$ such that $\rho(c)=\varphi_{1}(e)$. For the rest of this proof, we shall always be dealing with homology with local coefficients in $Z(G)$.

Some notation. The following denote inclusion maps:

$$
\begin{aligned}
& i:(\underbrace{\left.\tilde{A}^{\prime}, \tilde{A}\right)} \rightarrow(\tilde{X}, \tilde{A}) \\
& i^{\prime}:(\underbrace{\left(T\left(A^{\prime}\right)\right.}, \tilde{A}) \rightarrow(\tilde{X}, \tilde{A}) \\
& k:\left(T\left(A^{\prime}\right), \tilde{A}\right) \rightarrow\left(T\left(A^{\prime}\right), \tilde{A}^{\prime}\right) .
\end{aligned}
$$

$\partial$ denotes the boundary map in homology associated to the triple $\tilde{A} \widetilde{A^{\prime} \subset} \widetilde{X}$. $\partial^{\prime}$ denotes the boundary map associated to the triple $\tilde{A} \subset \widetilde{T\left(A^{\prime}\right)} \subset \tilde{X}$.

Remark. $t$ will be used to denote all maps between pairs of topological spaces induced by the covering transformation $t: \tilde{X} \rightarrow \tilde{X}$ as long as the context in which it is used makes its domain pair and range pair clear. The same remark applies to $t^{-1}$.

The first step in our proof of the inductive statement is the following.

Step $1^{\circ}$. There exists an element $x \varepsilon H_{s+1}\left(T\left(A^{\prime}\right), A\right)$ such that $i_{*}^{\prime}(x)=b$ and $t_{*}^{-1}(x)=c$.

Demonstration of step $1^{\circ}$. One can show by a short computation that there exists an $x^{\prime} \varepsilon H_{s+1}\left(T\left(A^{\prime}\right), A\right)$ such that $i_{*}^{\prime}\left(x^{\prime}\right)=b$. It is also easy to see that $i_{*}(c)=t_{*}^{-1}(b)=t_{*}^{-1} i_{*}^{\prime}\left(x^{\prime}\right)=i_{*} t_{*}^{-1}\left(x^{\prime}\right)$, and hence there exists $z \varepsilon H_{s+1}\left(X, A^{\prime}\right)$ such that $\partial z=c-t_{*}^{-1}\left(x^{\prime}\right)$. Let $z^{\prime}=t_{*}(z) \varepsilon H_{s+1}\left(X, T\left(A^{\prime}\right)\right)$. Let $x=\partial^{\prime}\left(z^{\prime}\right)+x^{\prime}$. Then, since $i_{*}^{\prime} \circ \partial^{\prime}=0$, we see that $i_{*}^{\prime}(x)=b$, and moreover that $t_{*}^{-1}(x)=$ $t_{*}^{-1}\left(x^{\prime}+\partial^{\prime}\left(z^{\prime}\right)\right)=t_{*}^{-1}\left(x^{\prime}\right)+t_{*}^{-1} \partial^{\prime}\left(z^{\prime}\right)$. Let us analyse $t_{*}^{-1} \partial^{\prime}\left(z^{\prime}\right)$. Since $t^{-1}:(\tilde{X}$, $\left.T\left(A^{\prime}\right), \tilde{A}\right) \rightarrow\left(\tilde{X}, \tilde{A}^{\prime}, \tilde{A}\right)$, we have that $t_{*}^{-1} \partial=\partial t_{*}^{-1}$, and hence $t_{*}^{-1} \partial^{\prime}\left(z^{\prime}\right)=$ $\partial t_{*}^{-1} t_{*}(z)=\partial z=c-t_{*}^{-1}\left(x^{\prime}\right)$. Therefore $t_{*}^{-1}(x)=t_{*}^{-1}\left(x^{\prime}\right)+\left(c-t_{*}^{-1}\left(x^{\prime}\right)\right)=c$, and this completes the demonstration of step $1^{\circ}$.

Let $y^{\prime}=k_{*}(x)$; then $y^{\prime} \varepsilon H_{s+1}\left(T\left(A^{\prime}\right), A^{\prime}\right)$. Let $W_{1}, W_{2}, \cdots, W_{i}$ denote the sequence of elementary cobordisms constructed so far, and let $\left(N_{i}, \nu_{i}\right)$ denote the right side of $W_{i}$. Then by considering the triple $\tilde{A} \subset \widetilde{A^{\prime}} \subset \tilde{X}$ we see that $\left(N_{i}, \nu_{i}\right)$ is an s-connected splitting of $M$. By applying the construction in the 
proof of Lemma 2.7 to the element $y^{\prime}$, we obtain an elementary cobordism $W_{i+1}$ of dimension $s+1$ with left side $\left(N_{i}, \nu_{i}\right)$, such that a generator of $H_{s+1}\left(W_{i+1}, N_{i}\right)$ maps onto $y^{\prime}$ under the inclusion map of $\left(\tilde{W}_{i+1}, \tilde{N}_{i}\right)$ into $\left(T\left(A^{\prime}\right), \tilde{A}^{\prime}\right)$. Let $y^{\prime \prime}$ denote this generator. (Here $W_{i+1}$ is used also to denote the lifting of $W_{i+1}$ to $X$ determined by the lifting of $N_{i}$ to $X$.) Let $A^{\prime \prime}=A^{\prime} \cup W_{i+1}$. (See Fig. 5.2).

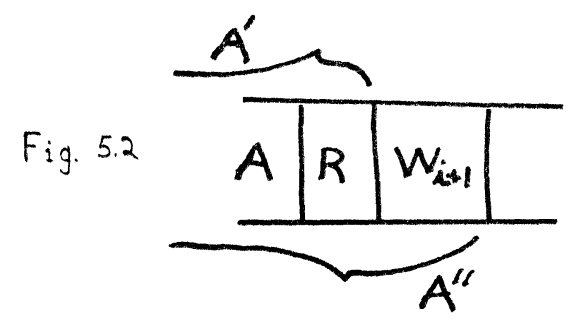

More notation. The following denote inclusion maps:

$$
\begin{aligned}
& I:\left(\widetilde{A^{\prime \prime}}, \tilde{A}\right) \rightarrow(\widetilde{X}, \tilde{A}) \\
& I^{\prime}:\left(\widetilde{A^{\prime \prime}}, \widetilde{A}\right) \rightarrow(\overbrace{\left(T\left(A^{\prime}\right), \tilde{A}\right)}^{\left(\widetilde{T}\left(A^{\prime}\right), \tilde{A}^{\prime}\right)} \\
& I^{\prime \prime}:\left(\widetilde{A^{\prime \prime}}, \tilde{A}^{\prime}\right) \rightarrow\left(\widetilde{A^{\prime \prime}}, \widetilde{A}^{\prime}\right) \\
& K:\left(\widetilde{A^{\prime \prime}}, \tilde{A}\right) \rightarrow\left(\widetilde{A^{\prime \prime}}, \tilde{A}\right) .
\end{aligned}
$$

To complete the proof of the inductive statement, we must construct a map $\rho^{\prime}: H_{s+1}\left(A^{\prime \prime}, A\right) \rightarrow P_{1}$ such that image $\rho^{\prime}$ is $F_{i+1}$ and $p \circ \rho^{\prime}=I_{*}$. The inclusion map of the triple $\left(A^{\prime \prime}, A^{\prime}, A\right)$ into the triple $\left(T\left(A^{\prime}\right), A^{\prime}, A\right)$ induces the following diagram in homology:

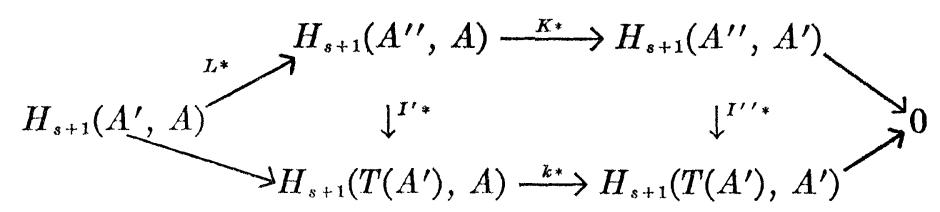

Using the facts that $I_{*}^{\prime \prime}\left(y^{\prime \prime}\right)=y^{\prime}$ and $k(x)=y^{\prime}$, we find, after chasing the above diagram, an element $y \varepsilon H_{s+1}\left(A^{\prime \prime}, A\right)$ such that $K_{*}(y)=y^{\prime \prime}$ and $I_{*}^{\prime}(y)=x$. Define $\rho: H_{s+1}\left(A^{\prime \prime}, A\right) \rightarrow P_{1}$ by letting $\rho^{\prime}(y)=e$ and $\rho^{\prime} \circ L_{*}=\rho$. This is well defined since $H_{*+1}\left(A^{\prime \prime}, A\right) \cong$ image $L_{*} \oplus\{y\}$ and $L_{*}$ is a monomorphism.

A) Demonstration that $p \circ \rho^{\prime}=I_{*}$. Notice that $p \rho^{\prime}(y)=p(e)=b=i_{*}^{\prime}(x)=$ $i_{*}^{\prime} I_{*}^{\prime}(y)=I_{*}(y)$. Also, if $u \varepsilon H_{*+1}\left(A^{\prime \prime}, A\right)$, such that $u=L_{*}(v)$ for some $v \varepsilon$ $H_{s+1}^{*}\left(A^{\prime}, A\right)$, then

$$
p \rho^{\prime}(u)=p \rho^{\prime} L_{*}(v)=p \rho(v)=i_{*}(v)=I_{*} L_{*}(v)=I_{*}(u) .
$$


It is also clear from its definition that image $\rho^{\prime}=F_{i+1}$. Hence it remains to prove the following.

B) $\rho^{\prime}$ is a map in $\mathrm{e}(Z(G), \alpha)$. Let $u=L_{*}(v)$. Then $\varphi_{1} \rho^{\prime}(u)=\varphi_{1} \rho(v)=\rho t_{*}^{-1}(v)=$ $\rho^{\prime} L_{*} t_{*}^{-1}(v)=\rho^{\prime}\left(t_{*}^{-1}\left(L_{*}(v)\right)\right)=\rho^{\prime}\left(t_{*}^{-1}(u)\right)$. Secondly, $\varphi_{1} \rho^{\prime}(y)=\varphi_{1}(e)=\rho(c)=$ $\rho^{\prime} L_{*}(c)=\rho^{\prime} L_{*} t_{*}^{-1}(x)$ (here we used step $1^{\circ}$ ). Since $I_{*}^{\prime}(y)=x, \varphi_{1} \rho^{\prime}(y)=\rho^{\prime} L_{*} t^{-1}$ $I_{*}^{\prime}(y)=\rho^{\prime} t_{*}^{-1}(y)$. This last step is a consequence of the following commutative diagram.

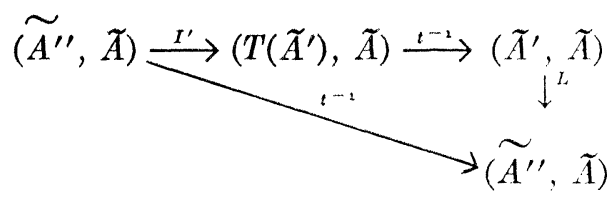

Hence, we have completed the proof of the inductive statement.

Chapter VI. The obstruction to a pseudo fibration fibering. In this chapter we show that if $M$ pseudo fibers a circle with respect to $f \varepsilon H^{1}(M ; Z)$, then there exists an obstruction $\tau(f)$ such that $\tau(f)$ vanishes if and only if there exists a smooth fiber map $\bar{f}: M \rightarrow S^{1}$ homotopic to $f$.

Assume that $c(f)=0$. Then there exists a splitting $(N, \nu)$ such that $W=$ $\overline{T(A)-A}$ is an $h$-cobordism $(W, N, T(N))$. By the $s$-cobordism theorem (see [8]), $W$ is diffeomorphic to $N \times[0,1]$ if and only if the Whitehead torsion $\tau(W, N)$ of the $h$-cobordism is the zero element of $W h(G)$. If this happens, then there exists a smooth fiber map $\bar{f}: M \rightarrow S^{1}$ such that $\bar{f}$ is homotopic to $f$ (see [3]). But it is possible that $\tau(W, N) \neq 0$ although there exists another splitting $\left(N^{\prime}, \nu^{\prime}\right)$ such that $\tau\left(W^{\prime}, N^{\prime}\right)=0$.

We proceed to measure this ambiguity. Let $\alpha_{*}: W h(G) \rightarrow W h(G)$ be the automorphism induced by $\alpha: Z(G) \rightarrow Z(G)$, and let $W h_{\alpha}(G)=W h(G) /\{x-$ $\left.\alpha_{*}(x) \mid x \varepsilon W h(G)\right\}$.

Definition 6.1. If $(N, \nu)$ is as above, we define $\tau(f) \varepsilon W h_{\alpha}(G)$ to be the image of $\tau(W, N)$ in $W h_{\alpha}(G)$.

Remark. $\quad \tau(f)$ is only defined when $c(f)=0$.

We proceed to show that $\tau(f)$ is well defined.

Step $1^{\circ}$. Since $T:(W, N) \rightarrow(T(W), T(N))$ is a diffeomorphism, we see easily that $\tau(T(W), T(N))=\alpha_{*}^{-1}(\tau(W, N))$. Therefore, if $p$ denotes the quotient map of $W h(G)$ onto $W h_{\alpha}(G)$, we see that $p(\tau(W, N))$ is independent of the lifting of $N$ to $X$.

Step $2^{\circ}$. Let $\left(N^{\prime}, \nu^{\prime}\right)$ be a second splitting such that $\left(W^{\prime}, N^{\prime}\right)$ is also an $h$ cobordism. By step $1^{\circ}$ we may assume that $T(A) \subset A^{\prime}$. Let $W^{\prime \prime}=\overline{A^{\prime}-T(A)}$. Then one sees easily that $\left(W^{\prime \prime}, T(N)\right)$ is an $h$-cobordism. Let $W_{1}=W \cup W^{\prime \prime}$ and $W_{2}=W^{\prime \prime} \cup W^{\prime}$ (see Fig. 6.1). 


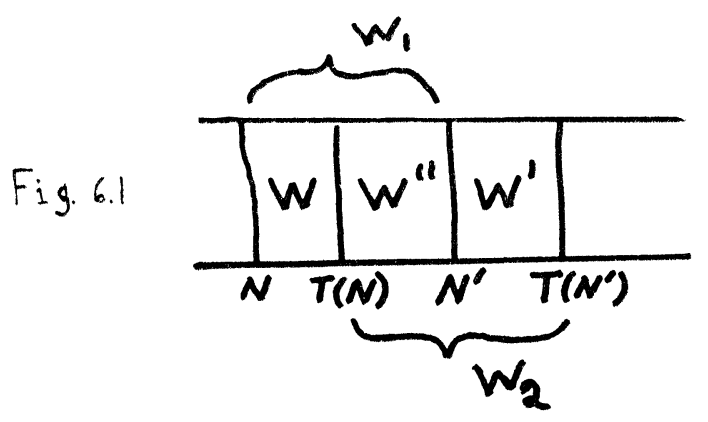

Since $T:\left(W_{1}, N\right) \rightarrow\left(W_{2}, T(N)\right)$ is a diffeomorphism, we see that $\tau\left(W_{2}, T(N)\right)$ $=\alpha_{*}^{-1} \tau\left(W_{1}, N\right)$. But $\tau\left(W_{1}, N\right)=\tau(W, N)+\tau\left(W^{\prime \prime}, T(N)\right)$, while $\tau\left(W_{2}\right.$, $T(N))=\tau\left(W^{\prime \prime}, T(N)\right)+\tau\left(W^{\prime}, N^{\prime}\right)$. Putting these three equations together, we see that $p(\tau(W, N))=p\left(\tau\left(W^{\prime}, N^{\prime}\right)\right)$, and hence $\tau(f)$ is well defined.

Theorem 6.2. When $c(f)=0$, there exists a smooth fiber map $\bar{f}: M \rightarrow S^{1}$ homotopic to $f$ if and only if $\tau(f)=0$.

The proof of Theorem 6.2 will depend on the following lemma.

Lemma 6.3. If $c(f)=0$ and $x \varepsilon p^{-1} \tau(f)\left(p: W h(G) \rightarrow W h_{\alpha}(G)\right)$, then there exists a splitting $\left(N^{\prime}, \nu^{\prime}\right)$ of $M$ such that $W^{\prime}=\overline{T\left(A^{\prime}\right)-A^{\prime}}$ is an h-cobordism and $\tau\left(W^{\prime}, N^{\prime}\right)=x$.

Proof of Lemma 6.3. Since $c(f)=0$, there exists a splitting $(N, \nu)$ such that $W=\overline{T(A)-A}$ is an $h$-cobordism. By definition, $p(\tau(W, N))=\tau(f)$, and hence $x=\tau(W, N)+y-\alpha_{*}(y)$ for some $y \varepsilon W h(G)$. By a result due to Stallings (see [13], page 398), there exists an $h$-cobordism $\left(W_{1}, N\right)$ such that $\tau\left(W_{1}, N\right)=$ $\alpha_{*}(y)$. Let $N^{\prime}$ be the right side of $W_{1}$. Then there exists a second $h$-cobordism $\left(W_{2}, N^{\prime}\right)$ such that $\tau\left(W_{2}, N^{\prime}\right)=-\alpha_{*}(y)$. We can identify half of a narrow tubular $n b d$. of $N$ with $W_{1} \cup W_{2}$. (see Fig. 6.2).
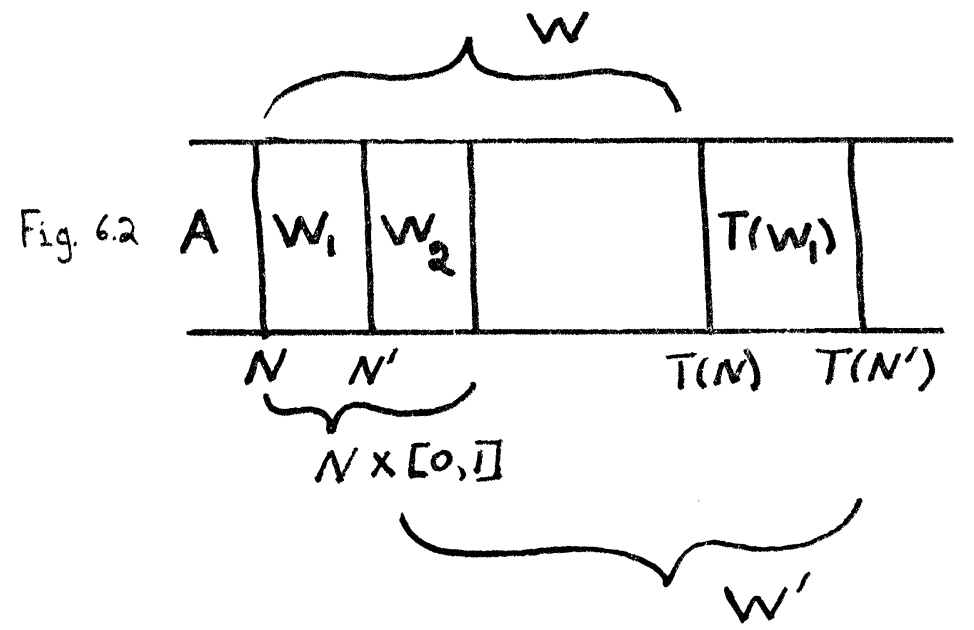
Let $A^{\prime}=A \cup W_{1}$ and $W^{\prime}=\overline{T\left(A^{\prime}\right)-A^{\prime}}$. Then $\tau\left(W_{1} \cup W^{\prime}, N\right)=\tau(W \cup$ $\left.T\left(W_{1}\right), N\right)$. But $\tau\left(W_{1} \cup W^{\prime}, N\right)=\alpha_{*}(y)+\tau\left(W^{\prime}, N^{\prime}\right)$, while $\tau\left(W \cup T\left(W_{1}\right), N\right)$ $=\tau(W, N)+\alpha_{*}^{-1} \alpha_{*}(y)$, and hence $\tau\left(W^{\prime}, N^{\prime}\right)=x$, where $\left(N^{\prime}, \nu^{\prime}\right)$ is a splitting relative to $f$.

Proof of Theorem 6.2.

Step $1^{\circ}$. If a smooth fiber map $\bar{f}: M \rightarrow S^{1}$ exists, let $N$ denote a fiber of this map. Then clearly $W=\overline{T(A)-A}$ is diffeomorphic to $N \times[0,1]$, and hence $\tau(W, N)=0$. Therefore, $\tau(f)=0$.

Step $2^{\circ}$. If $\tau(f)=0$, then by Lemma 6.3 there exists a splitting $(N, \nu)$ of $M$ such that $\tau(W, N)=0$. Hence, by the $s$-cobordism theorem (see [8]), $W$ is diffeomorphic to $N \times[0,1]$, and therefore, by [3], there exists a smooth fiber map $\bar{f}: M \rightarrow S^{1}$ homotopic to $f$.

This completes the proof of our main theorem.

Theorem 6.4. An indivisible element $f \varepsilon H^{1}(M ; Z)$ is homotopic to a smooth fiber map $\bar{f}: M \rightarrow S^{1}$ if and only if

$1^{\circ} X$ is the homotopy type of a finite C. W. complex.

$$
\begin{aligned}
& 2^{\circ} c(f)=0 . \\
& 3^{\circ} \tau(f)=0 .
\end{aligned}
$$

\section{REFERENCES}

1. H. Bass, A. Heller, \& R. Swan, The Whitehead group of a polynomial extension, Publ. de l'Inst. des Hautes Etude Sci. \#22.

2. L. Siebenmann, The Obstruction to finding a boundary for an open manifold of dimension greater than five, Ph.D. thesis, Princeton University.

3. W. Browder \& J. Levine, Fibering manifolds over a circle, Comment. Math. Helv. 40 (1966), 153-160.

4. C. T. C. WALL, Differential topology, mimeographed notes, Cambridge University.

5. I. Berstein \& C. Thomas, Structures on $M \times \mathrm{R}$ in the non-simply connected case, mimeographed, Cornell University.

6. C. T. C. WALL, Finiteness conditions for C. W. complexes, Ann. of Math. 81, 56-59.

7. W. Browder, Structures on $M \times R$, Proc. Camb. Phil. Soc. 61 (1965), 337-345.

8. D. Barden, Structure of manifolds, Ph.D. thesis, Cambridge University.

9. C. T. C. WALL, Surgery of non-simply connected manifolds, Ann. of Math. 84, 217-276.

10. W. Browder, Cap products and Poincaré duality, mimeographed notes, Cambridge University.

11. J. Milnor, The theory of characteristic classes, appendix A, mimeographed notes, Princeton University.

12. J. Milnor, Lectures on the h-cobordism theorem, Princeton Mathematical Notes, Princeton University Press.

13. J. Milnor, Whitehead Torsion, Bull. Amer. Math. Soc. 72, 358-426.

14. H. BAss \& M. P. MURPHY, Grothendieck groups and Picard groups of abelian group rings, Ann. of Math. 86, 16-73. 
15. K. W. Kwan \& R. H. Szczarba, Product and sum theorems for Whitehead torsion, Ann. of Math. 82, 183-190.

16. J. Stallings, On fibering certain 3-manifolds, Topology of 3-manifolds, Prentice-Hall (1962), 95-100.

17. F. T. FARrell, The obstruction to fibering a manifold over a circle, Bull. Amer. Math. Soc. 73, 737-740.

18. F. T. Farrell \& W. C. Hsiang, H-Cobordant manifolds are not necessarily homemorphic, Bull. Amer. Math. Soc. 73, 741-744.

19. F. T. FArrell \& W. C. Hsiang, A geometric interpretation of the Künneth formula for algebraic K-theory, (to appear).

20. J. Stallings, On infinite processes leading to differentiability in the complement of a point, Differential and Combinatorial Topology, (A Syposium in honor of M. Moore), Princeton University Press, Princeton, N. J., 245-254.

21. F. T. FARRell, The obstruction to fibering a manifold over a circle, Ph.D. thesis, Yale University, (1967), obtainable from University Microfilms, Ann Arbor, Michigan.

22. F. T. FARRELL, The obstruction to fibering a manifold over a circle, Proceedings of the International Congress of Mathematicians, Nice, France (1970).

23. F. T. Farrell \& W. C. Hsiang, A formula for $\mathrm{K}_{1} R_{\alpha}[\mathrm{T}]$, Proceedings of Symposia in Pure Mathematics XVII (1970), 192-218.

University of California

Berkeley

Date communicated: MAY 20, 1971 